\title{
Turning intractable counting into sampling: computing the configurational entropy of three-dimensional jammed packings
}

\author{
Stefano Martiniani, ${ }^{1, *}$ K. Julian Schrenk, ${ }^{1, \dagger}$ Jacob D. Stevenson, ${ }^{2,1}$ David J. Wales, ${ }^{1}$ and Daan Frenkel ${ }^{1}$ \\ ${ }^{1}$ Department of Chemistry, University of Cambridge, Lensfield Road, Cambridge, CB2 1EW, UK \\ ${ }^{2}$ Microsoft Research Ltd, 21 Station Road, Cambridge, CB1 2FB, UK
}

\begin{abstract}
We report the first numerical calculation of the total number of disordered jammed configurations $\Omega$ of $N$ repulsive, three-dimensional spheres in a fixed volume $V$. To make these calculations tractable, we increase the computational efficiency of the approach of Xu et al. (Phys. Rev. Lett. 106, 245502 (2011)) and Asenjo et al. (Phys. Rev. Lett. 112, 098002 (2014)) and we extend the method to allow computation of the configurational entropy as a function of pressure. The approach that we use computes the configurational entropy by sampling the absolute volume of basins of attraction of the stable packings in the potential energy landscape. We find a surprisingly strong correlation between the pressure of a configuration and the volume of its basin of attraction in the potential energy landscape. This relation is well described by a power law. Our methodology to compute the number of minima in the potential energy landscape should be applicable to a wide range of other enumeration problems in statistical physics, string theory, cosmology and machine learning, that aim to find the distribution of the extrema of a scalar cost function that depends on many degrees of freedom.
\end{abstract}

\section{INTRODUCTION}

Many questions in physics are easy to pose but difficult to answer. One such question is: how many microscopic states of a given system are compatible with its macroscopic properties? In statistical mechanics, knowledge of this number allows us to compute the entropy, and thereby predict the macroscopic properties of a system from knowledge of the interaction between atoms or molecules.

In granular matter we can similarly ask how many microstates are compatible with a given set of macroscopic properties. However, the computation of the corresponding absolute entropy has thus far proven to be extremely challenging. Without such knowledge, it is not possible to explore the analogies and differences between granular and Boltzmann entropy. Being able to compute the configurational entropy is therefore clearly important. The more so as granular materials are ubiquitous in everyday life (sand, soil, powders). Many industrial processes involve granular materials. In the natural world, the Earth's surface contains vast granular assemblies such as dunes, which interact with wind, water, and vegetation [1]. Packings of particles that are soft or biological in nature, such as cells, hydrogels and foams are also known to undergo jamming [2] and their behaviour to be "granular" viz not subject to thermal motion. Moreover, as glasses and granular materials share many properties it has been proposed that their physics may be controlled by the same underlying principles [3].

The study of granular materials is complicated by the fact that these materials are intrinsically out-ofequilibrium. In fact, thermal motion plays no role in

\footnotetext{
*sm958@cam.ac.uk

$\dagger$ kjs73@cam.ac.uk
}

granular matter. It maintains its configuration unless driven by external forces. As a consequence, the properties of granular materials depend upon their preparation protocol.

Granular materials are athermal and cannot therefore be described by statistical mechanics. However, these materials can exists in a very large number of distinct states and this fact inspired Edwards and Oakeshott [4] well over two decades ago to propose a statisticalmechanics-like formalism to describe the properties of granular matter. In its original version, the Edwards theory assumed that all mechanically stable configurations ('jammed' states) are equally probable and that the logarithm of the number of these states plays a role similar to that of entropy. In this theoretical framework, the volume of the system and its compactivity (i.e. the derivative of volume with respect to the configurational entropy) are the analogues of the energy and temperature in thermal systems.

In the absence of explicit calculations (or measurements) of the absolute configurational entropy, a direct test of the Edwards hypothesis has proven difficult, and different authors have arrived at different conclusions based on indirect tests in either simulations [5-8] or experiments $[9,10]$. In addition, alternative definitions of entropy have been proposed to characterise the complexity of granular systems while circumventing explicit enumeration of states $[11,12]$.

Numerous tests of the Edwards volume ensemble have focused on the determination of the compactivity [13-21]. However, the role of compactivity as a temperature-like quantity is problematic as Puckett and Daniels [22, 23] have shown that it does not satisfy the equivalent of the zero-th law of Thermodynamics - the law that is the basis of all thermometry.

Edwards' theory has been generalised to include the distribution of stresses within the system through the force-moment tensor [24-27] and another analogue of 
temperature emerged, known as angoricity, which is a measure of the change in entropy with stress. The experiments by Puckett and Daniels [22] showed that angoricity, unlike compactivity, is a temperature-like quantity as it satisfies the zero-th law.

To date only a few examples of numerical tests of the generalised Edwards ensemble are available [22, 26, 28, 29]. Numerical tests of the stress ensemble focus on systems of soft spheres near jamming where the compactivity $X \rightarrow \infty$ and fluctuations in volume are negligible compared to stress fluctuations [26, 27]. Wang et al. $[28,29]$ proposed a unified test that compared ensemble averaged results over volume and stress with predictions for the jamming transition, finding agreement; we note, however, that in the latter approach the results rely significantly on the equiprobability assumption.

When the system is composed of very stiff grains, or is close to jamming, any small deformation will lead to a large change in the contact forces. In these limits the geometric and the force degrees of freedom can be decoupled, giving rise to the force network ensemble [30] (FNE). In this framework, force networks are constructed on a given geometry and each force state is assumed to be equiprobable. The FNE has been utilised as a testing ground for statistical frameworks [31-33].

More than two decades after its introduction many fundamental questions concerning the Edwards hypothesis remain unanswered. This unsatisfactory state of affairs is at least partly due to the fact that no efficient methods existed to measure or compute the absolute configurational entropy directly. Until recently, the only way to determine the configurational entropy was by direct enumeration of the distinct jammed states of a system. This method is inefficient and cannot be used for systems that contain more than 10-20 particles. Over the past few years, the situation on the numerical front has changed: recent numerical work by Asenjo et al. [34, 35], based on an approach introduced by $\mathrm{Xu}$ et al. [36], has demonstrated that it is possible to compute the number of distinct jammed states of a system, even when this number is far too large (e.g. $10^{250}$ ) to allow direct enumeration. The approach of Refs. [34-36] replaces an intractable enumeration problem by a tractable scheme to sample the (absolute) volume of the basins of attraction of stable states in the potential energy landscape.

The approach described herein is completely general and it extends to any energy landscape problem that aims to find the extrema of a scalar cost function that depends on many degrees of freedom. Enumerating the number of solutions or stationary points, and their distribution, for certain classes of random functions is a classical problem in mathematics and statistics [37-50]. In statistical physics, ad hoc numerical and theoretical methods have been developed in the realms of random Gaussian and polynomial fields [51-59]. In this sense, particular attention has been devoted to the mean-field p-spin spherical model of a spin glass with quenched disorder [60-64]. A related area is the computation of the configurational

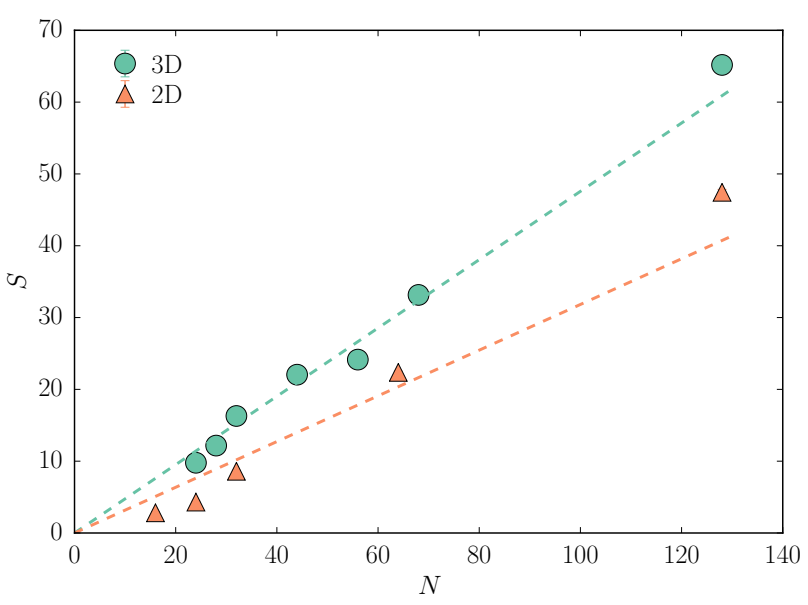

FIG. 1: Entropy as a function of system size $N$ for two (Ref. [34]) and three-dimensional (this work) jammed sphere packings. Dashed curves are lines of best fit of the form $S=a N$.

contribution to the entropy of structural glasses $[65,66]$. The physical significance of this method goes even further to encompass string theory [67-69], cosmology [7074] and machine learning [75-78].

We note that the geometrical structure of the basins of attraction of jammed states had been studied by O'Hern and co-workers $[8,79,80]$. O'Hern also reported direct enumeration estimates of the number of jammed states of small systems. A rather different technique ('basin sampling') to count the number of energy minima in the potential energy landscape of small clusters had been reported by Wales and co-workers [81, 82].

We note that, for the system (and protocol) considered by Asenjo et al., not all packings are equally probable. However, as shown in Ref. [34], the equal-probability hypothesis is not needed to arrive at a meaningful definition of an extensive granular entropy. When, in the remainder of the present paper, we mention the configurational (granular) entropy, we refer to the definition of Ref. [34].

We stress that, even though the approach of Refs. [3436] allows to solve enumeration problems which were far from possible using direct enumeration, it is still computationally expensive. Thus far, it had only been applied to two-dimensional packings. Substantial 'technical' improvements were needed to make the method fast enough to deal with three-dimensional systems.

In the present paper, we present the first enumeration of the number of jammed packings for three-dimensional systems consisting of up to 128 soft spheres. A direct comparison of the entropy measured as a function of system size for two and three-dimensional jammed sphere packings is shown in Fig. 1. The potential of the method presented herein can be verified unequivocally from Fig. 1: we are able of tackling problems at least 500 million times more complex, and of greater computa- 
tional cost, than the already spectacularly difficult questions confronted by Asenjo et al. [34]. Furthermore we show how our improved procedure allows first-principles computation of configurational entropy as a function of system size and pressure. The method and the technical improvements needed to overcome this numerical challenge are presented alongside the main results.

The remainder of this work is organised as follows. Section II describes the basic principle of the mean basin volume method for counting, and explains how that strategy can be applied to enumerate granular packings. The enumeration and entropy results for three-dimensional jammed sphere packings as a function of system size and pressure are reported in Sec. III. The rest of the manuscript is dedicated to the description of the improved numerical method. Section IV outlines our protocol for sampling different granular packings, and it describes the corresponding potential energy landscape and minimisation techniques. Application of thermodynamic integration to compute the volume of a basin of attraction in such a landscape is described in Sec. V. Aspects of the data analysis tools used on the histograms of sampled basin volumes, and related configurational entropy definitions, are described in Sec. VI. Conclusions are drawn in Sec. VII. Further technical background is given in the appendices.

\section{BASIC PRINCIPLE: COUNTING BY SAMPLING}

In this section, we briefly review the numerical approach that we use to compute the number of distinct jammed states. We stress that the approach that we use has much wider applicability than the counting of granular packings [51-68, 70-78, 83]. In the context of granular packings, our aim is to compute the number of ways $\Omega$ in which $N$ spheres can be arranged in a given volume $V_{\text {box }}$ of Euclidean dimension $d$. Knowledge of $\Omega$ allows us to compute configurational entropies and related quantities from first principles [4, 34]. Our approach is based on a rigorous mapping of the enumeration problem onto counting the number of minima of a potential energy landscape [36]. The approach makes no use of a harmonic [84] or quasi-harmonic [85] approximation. For a system of hard particles the potential energy function is discontinuous, that is, the energy of the system is either zero, if no two particles overlap, or infinity otherwise. Then, at jamming, in the absence of rattlers, basins of attraction are single points in configuration space and they have no associated volume. This does not mean that we cannot sample the energy minima of a system of hard particles. The reason is that all jammed structures of hard particles correspond to the zero potential energy minima of a system with a continuous repulsive potential with the same range as the hard-core diameter of the hard particles. In what follows, we focus on this class of systems, but we generalise the problem by also considering minima with a non-zero potential energy. In particular, we consider spherical particles with a hard core and a short-ranged continuous repulsive interaction. Under conditions where this system is jammed, a system with only the hard-core interactions would still be fluid and would sample the accessible configuration space uniformly. This remaining accessible volume is partitioned in basins of attraction defined by the soft shells. The HS-WCA potential used to simulate hard-core plus softshell interactions and the packing preparation protocol are described in Sec IV B. For an illustration of the packing preparation protocol refer to Fig. 2. As we argue below, using an HS-WCA model greatly improves the efficiency of determination of basin volumes.

Let us denote the total available volume in $d N$ dimensional space as $\mathcal{V}$. Note that $\mathcal{V}$ is not the total volume of configuration space $\left(V^{N}\right)$, but just that part of the volume that is free of hard-core overlaps. It is the configurational part of the partition function of the hard-core system at the number density under consideration. Since the accessible configuration space is tiled by the basins of attraction of the distinct energy minima [84, 86-88] we can write:

$$
\mathcal{V}=\sum_{i=1}^{\Omega} v_{i}
$$

where $v_{i}$ is the volume of the $i$-th basin of attraction and $\Omega$ is the total number of distinct minima. We thus make the simple observation:

$$
\sum_{i=1}^{\Omega} v_{i}=\frac{\Omega}{\Omega} \sum_{i=1}^{\Omega} v_{i}=\Omega\langle v\rangle,
$$

where $\langle v\rangle$ is the mean basin volume, from which it follows immediately that

$$
\Omega=\frac{\mathcal{V}}{\langle v\rangle}
$$

We note that, for sphere packings, $\mathcal{V}$ is known from the equation of state of the underlying hard sphere fluid (see Appendix E) and we can measure $\langle v\rangle$ by thermodynamic integration, as discussed in detail in the Sec IV. The approach of $[34,36]$ has thus turned the intractable enumeration problem of finding $\Omega$ into a sampling one, namely measuring $\langle v\rangle$.

\section{RESULTS: COUNTING DISORDERED 3D SPHERE PACKINGS}

The mean basin volume method for enumerating the number of mechanically stable packings was introduced by $\mathrm{Xu}$ et al. [36], and tested on a small system of soft disks. Asenjo et al. [34] then made a number of modifications to the algorithm that allowed them to apply it to larger systems of up to 128 disks. As was the case with 

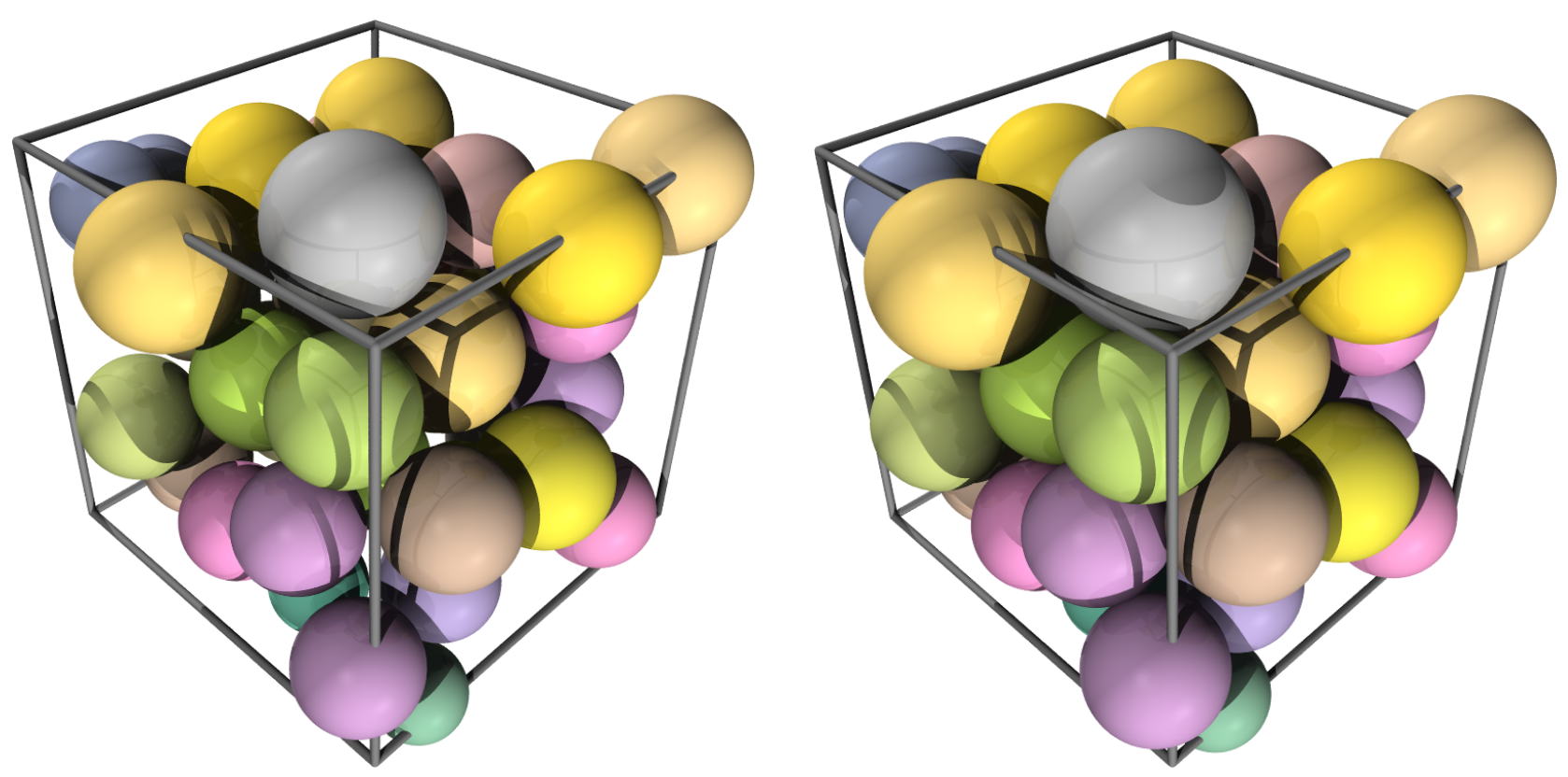

FIG. 2: Hard sphere fluid at $\phi_{\mathrm{HS}}=0.5$, left, and HS-WCA jammed packing at $\phi_{\mathrm{SS}}=0.7$, right, for a system of 44 polydisperse hard spheres with mean radius $\left\langle r_{h}\right\rangle=1$ and standard deviation $\sigma_{\mathrm{HS}}=0.05$. We prepare the polydisperse HS fluid configurations at fixed packing fraction $\phi_{\mathrm{HS}}=0.5$ by a Monte Carlo simulation. Particles are then inflated by the same factor, proportional to their radius (spheres are coloured according to their radius), to obtain an over-compressed soft spheres jammed packing at $\phi_{\mathrm{SS}}=0.7$ by an infinitely fast quench (energy minimisation).

Ref. [36], the calculations of Ref. [34] focused on twodimensional systems because of the high computational costs involved in studying 3D systems. Here we present results for systems of three-dimensional soft spheres. We are thus in a position to compute the configurational entropy of a real (but idealised) three-dimensional system.

We first describe an analysis similar to the one reported by Asenjo et al. [34] to verify the extensivity of the entropy $S(V)$ at constant packing fraction. Next, we extend our approach to the generalised Edwards ensemble, i.e. one based on a description of the system in terms of its volume and pressure, to compute the generalised entropy $S(V, \mathcal{P})$.

We investigate three-dimensional packings with system sizes ranging from 24 to 128 HS-WCA particles, see Eq. (15), at $\phi_{\mathrm{HS}}=0.5$ hard-sphere fluid packing fraction and $\phi_{\mathrm{SS}}=0.7$ soft sphere packing fraction, corresponding to a ratio of the soft and hard-sphere radii ratio $r_{\mathrm{SS}} / r_{\mathrm{HS}}=1.12$, prepared following the protocol outlined in Sec. IV. For each system size we compute the volume of the basin of attraction of approximately 1000 packings. Each PT run (see Sec. V) was performed on 15 parallel threads of a single eight-core dual Xeon E5 - 2670 $(2.6 \mathrm{GHz}$, Westmere) node. Our choice of convergence criterion was such that when the uncorrelated statistical error for each of the replicas' mean square displacement fell below $5 \%$ the calculations were terminated. This setup translated in run times ranging from 10 to 300 hours per basin depending on system size, which amounts to $\mathcal{O}\left(10^{6}\right)$ hours of total run time and $\mathcal{O}\left(10^{7}\right)$ total cpu hours. We then analyse the corresponding distribution of dimensionless free energies following the protocol described in Sec. V and VI and summarised in Appendix A.

\section{A. Extensivity of the entropy}

We first computed two alternative definitions of entropy: the Gibbs entropy $S_{G}=-\sum_{i=1}^{\Omega} p_{i} \ln \left(p_{i}\right)-\ln (N !)$ and Edwards (Boltzmann) entropy $S_{B}=\ln (\Omega)-\ln (N !)$, where $p_{i}$ is the probability to sample packing $i$ and $\Omega$ is the total number of mechanically stable states (or minima in the energy landscape). A detailed discussion of these definitions is outlined in Sec. VI. The results of these calculations are summarised in Fig. 3. Our results strongly suggest that, also in three dimensions, the entropy thus defined is extensive. Note that extensivity requires not only that the entropy scales linearly with system size, but also that it crosses zero at the origin. The slightly higher value of the Edwards entropy compared to the Gibbs entropy is consistent with the observation that Edwards' equiprobability corresponds to the maximum possible entropy of a system with $\Omega$ states. We also show that our estimates for the Edwards' entropy are relatively insensitive to the precise strategy used to compute it. In Fig. 3, we compare three methods: a parametric fit to a generalised Gaussian cumulative distribution function (c.d.f.) using a non-linear least squares method, a fit to the corresponding probability density function (p.d.f.) using maximum likelihood, and a non-parametric fit by 

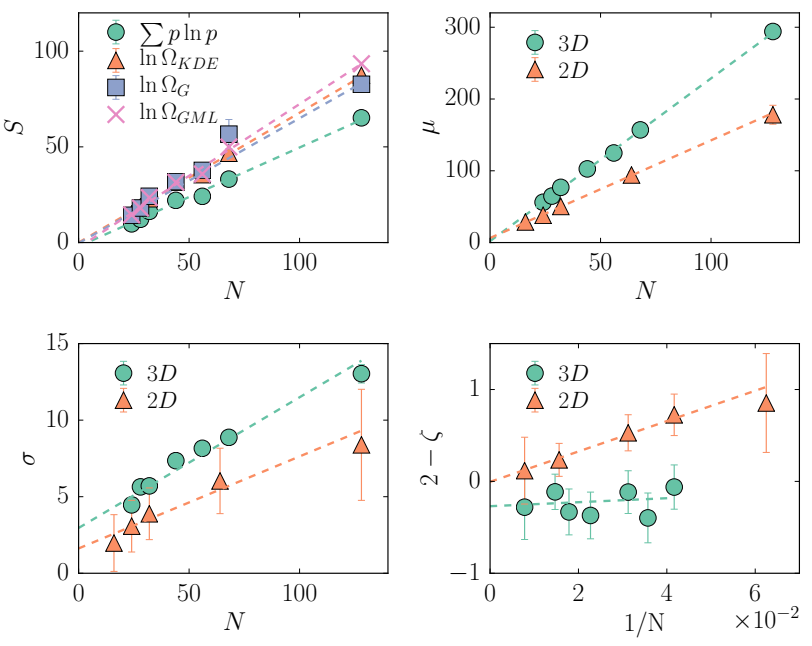

FIG. 3: Top left, entropy as a function of the system size $N$ computed, in order, according to the Gibbs configurational entropy and the Edwards configurational entropy using a non-parametric fit by kernel density estimation (KDE), a parametric fit to a generalised Gaussian c.d.f. using a non-linear least squares method and a fit to the corresponding p.d.f. using maximum likelihood (ML). Comparison of generalised Gaussian best-fit parameters for 2D (see Ref. [34]) and 3D sphere packings: scale parameter $\sigma$ (bottom left) and mean log-volume $\mu$ (top right) scale linearly with system size $N$; distributions are more peaked for $2 \mathrm{D}$ packings. In $2 \mathrm{D}$ we observe much stronger dependence of the shape parameter $\zeta$ (bottom right) as a function of system size than in $3 \mathrm{D}$.

kernel density estimation, which makes no a priori assumption about the shape of the distribution, other than the choice of the kernel function. We note, once again, that no post-processing is needed to compute the Gibbs version of the configurational entropy. Our results are in line with those reported by Asenjo et al. [34] for twodimensional systems.

The number of mechanically stable states $\Omega$ required by the Edwards' definition of entropy is obtained subsequently to fitting the numerically obtained distribution of log-basin volumes (dimensionless free energies) to a generalised Gaussian distribution and unbiasing it appropriately, as described in Sec VI. We observe that the best-fit mean and scale parameters of the generalised Gaussian for the distribution of dimensionless free energies, $\mu$ and $\sigma$ in Eq. (27) respectively, are also extensive, which although in line with what was found in two dimensions, is not a priori obvious. Finally we find that the shape parameter, $\zeta$ in Eq. (27), appears to depend only weakly on system size. The statistics are poor, but the data are compatible with the assumption that $\zeta \rightarrow 2$ (Gaussian distribution) as $N \rightarrow \infty$. In $2 \mathrm{D}$, the same limiting distribution of $\zeta$, but with a much stronger size dependence, was observed.

\section{B. Entropy in the generalised Edwards ensemble}

We next consider the situation where the configurational entropy is a function of both the volume $V$ and the stress tensor $\hat{\Sigma}$ of the system. The number of packings with fixed $V$ and $\hat{\Sigma}$ is denoted by $\Omega(\hat{\Sigma}, V)$.

In the generalised Edwards ensemble [23, 26, 27], we fix the variables conjugate to $V$ and $\hat{\Sigma}$, viz. the compactivity $X$ and the inverse angoricity tensor $\hat{\alpha}$. The generalised 'partition function' can then be written as [23]:

$$
Z_{\mathrm{dyn}}=\sum_{\nu} \omega\left(\hat{\Sigma}_{\nu}, V_{\nu}\right) e^{-V_{\nu} / X} e^{-\operatorname{Tr}\left(\hat{\alpha} \hat{\Sigma}_{\nu}\right)},
$$

where $V_{\nu}$ and $\hat{\Sigma}_{\nu}$ are the volume and the force-moment (stress) tensor for state $\nu$. The weights $\omega$ account for the protocol dependence of the probability to generate a state, and the sum runs over all mechanically stable states $\nu$.

We can rewrite this partition function in terms of the density of states:

$$
Z_{\mathrm{dyn}}=\prod_{l, k>l} \iint \mathrm{d} \hat{\Sigma}^{l k} \mathrm{~d} V \Omega_{\mathrm{dyn}}(\hat{\Sigma}, V) e^{-V / X} e^{-\operatorname{Tr}(\hat{\alpha} \hat{\Sigma})} .
$$

For a system under hydrostatic pressure, and in the absence of shear, we can write the force-moment tensor as $\hat{\Sigma}=\hat{I} \Gamma$, where $\Gamma=\mathcal{P} V=\operatorname{Tr}(\hat{\Sigma}) / 3$ is the internal Virial of the system. The inverse angoricity tensor $\hat{\alpha}$ becomes a scalar $\alpha=\partial S / \partial \Gamma$ [27]. This result allows to simplify the notation significantly and at fixed volume, through the mean basin volume method, we obtain the number of states integrated over all pressure states, $\Omega(V)=\int \mathrm{d} \mathcal{P} \Omega(V, \mathcal{P})$. We now discuss how to generalise this procedure so that one can compute $\Omega(V, \mathcal{P})$, and therefore the configurational entropy, in the context of the generalised Edwards ensemble.

\section{Pressure to basin volume power-law relation}

To compute $Z(X, \alpha)$ directly, we would have to evaluate $\Omega(\mathcal{P}, V)$ as a function of both $\mathcal{P}$ and $V$. Whilst, with the tools that we have, this calculation is in principle possible, the computational costs would be several orders of magnitude larger than the, already quite substantial, costs of computing $\Omega(V)$. This would suggest that the computation of $Z(X, \alpha)$ is not possible at present.

However, it turns out that we can still estimate the generalised configurational entropy because, as we discuss below, we observe a surprisingly strong correlation between pressure and basin volume.

From Fig. 4 we see that the basin volume for a given pressure state at fixed volume is strongly correlated with the pressure $\mathcal{P}$. As the figure suggests, the relation between $-\ln (v)$ and $\ln (\mathcal{P})$ is approximately linear, and hence

$$
F\left(\mathcal{P} \mid N, \phi_{\mathrm{SS}}\right) \equiv-\ln (v)=\frac{N}{\kappa} \ln (\mathcal{P})+\mathcal{C}(N),
$$



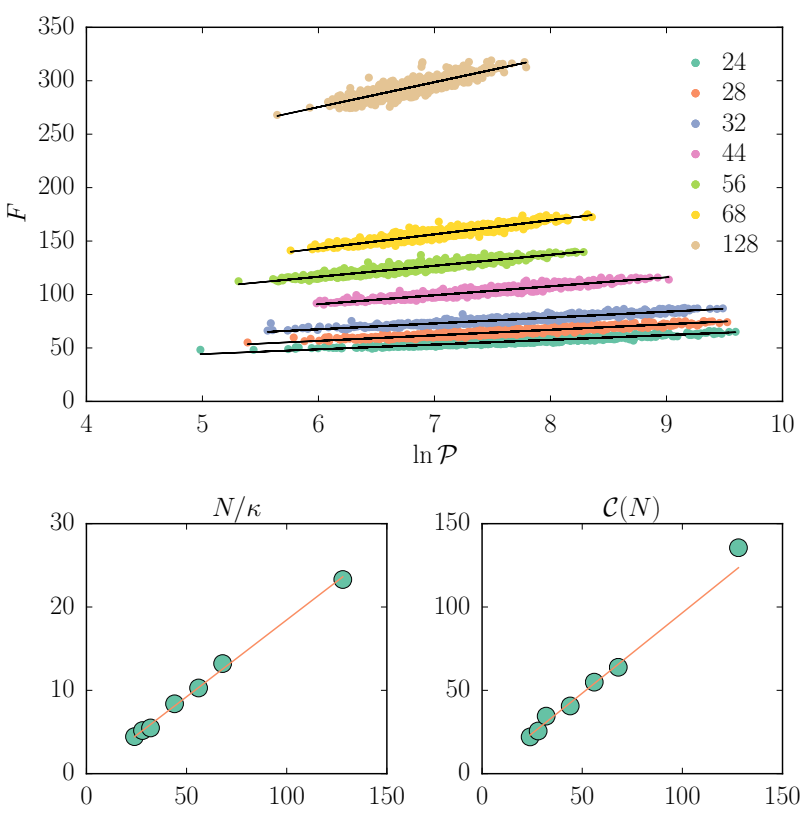

FIG. 4: Top: dimensionless free energy versus pressure of mechanically stable states at fixed volume for several system sizes. Best fit lines are in black. In the bottom left and right plots we show slope and intercept for each of the best fit lines as a function of system size. Both

slope and intercept scale linearly with system size.

where $\kappa$ denotes the slope of the linear fit, and $\mathcal{C}(N)$ its value at $\mathcal{P}=1$ (see Fig. 4 ). The value of $\kappa$ is not known a priori. It seems likely that $\kappa$ depends on the functional form of the potential. $\mathcal{C}(N)$ is a even less universal linear function of $N$, as it depends on the choice of units.

We anticipate that this power law relationship survives for packings in two dimensions for a wide spectrum of packing fractions $\phi>\phi_{J}$ viz. as long as the system is jammed and sufficiently over-compressed [89].

\section{Gibbs configurational entropy}

Using our approximate relation between pressure and basin volume, we can now rewrite Eq. (6) in terms of the probabilities for each jammed state

$$
\ln \left(p_{i}\right)=-\frac{N}{\kappa} \ln \left(\mathcal{P}_{i}\right)-\mathcal{C}(N)-\ln (\mathcal{V}),
$$

which when substituted in the definition for the Gibbs entropy Eq. (22), gives the configurational entropy at a given volume in terms of the biased mean log-pressure

$$
S_{G}=\frac{N}{\kappa}\langle\ln (\mathcal{P})\rangle_{\mathcal{B}}+\mathcal{C}(N)+\ln (\mathcal{V})-\ln (N !)
$$

The significance of this equation should be apparent: for a sufficiently over-compressed packings of soft spheres at

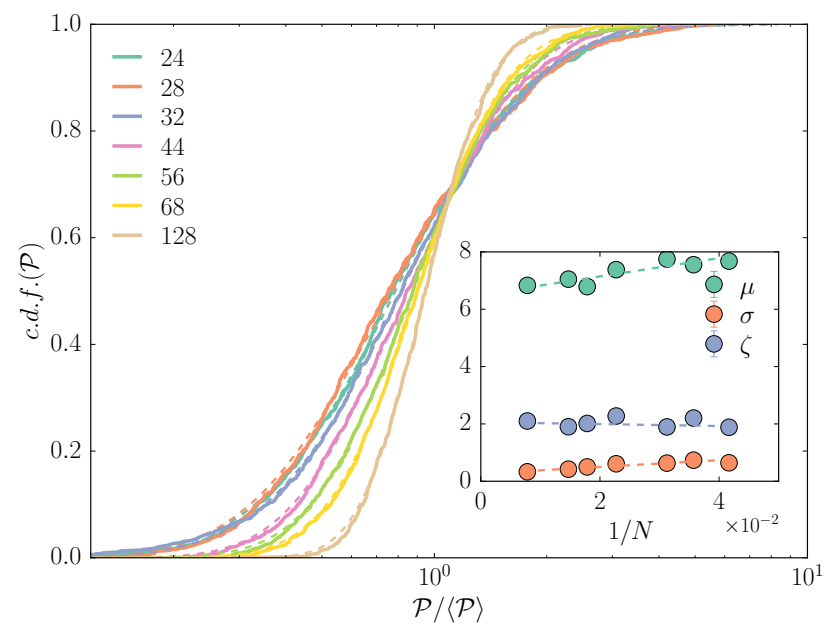

FIG. 5: Empirical cumulative distribution functions of the pressures for several system sizes. Dashed lines in the corresponding colour are curves of best fit to a generalised log-normal distribution. The curves are mostly indistinguishable. Inset: best fit parameters for the generalised log-normal distribution as a function of system size. The mean $\mu$ and scale parameter $\sigma$ scale linearly with $1 / N$, while the shape parameter $\zeta$ is approximately insensitive with respect to system size.

a given packing fraction, the Gibbs configurational entropy can be approximately computed from sole knowledge of the average pressure, provided that $\kappa$ is known.

\section{Generalised Edwards configurational entropy}

To recover the number of states as a function of volume and stress we note that

$$
\Omega(V, \mathcal{P})=\Omega(V) \int_{\mathcal{P}}^{\mathcal{P}+\delta \mathcal{P}} \mathcal{U}(x \mid V) \mathrm{d} x
$$

where $\mathcal{U}(\mathcal{P} \mid V)$ is the unbiased probability distribution function of stresses at some specified volume. The directly measured distribution of pressures depends on the protocol with which packings are generated.

We distinguish between the biased, $\mathcal{B}\left(\mathcal{P} \mid N, \phi_{\mathrm{SS}}\right.$ ) (as sampled by the packing protocol), and the unbiased, $\mathcal{U}\left(\mathcal{P} \mid N, \phi_{\mathrm{SS}}\right)$, pressure distributions. Since the configurations were sampled proportional to the volume of their basin of attraction, using Eq. (6) we can compute the unbiased distribution analogously to Eq. (25) as

$$
\mathcal{U}\left(\mathcal{P} \mid N, \phi_{\mathrm{SS}}\right)=\mathcal{Q}\left(N, \phi_{\mathrm{SS}}\right) \mathcal{B}\left(\mathcal{P} \mid N, \phi_{\mathrm{SS}}\right) e^{\mathcal{C}(N)} \mathcal{P}^{N / \kappa}
$$

where $\mathcal{Q}\left(N, \phi_{\mathrm{SS}}\right)=\langle v\rangle\left(N, \phi_{\mathrm{SS}}\right)$ is the normalisation constant.

Upon substitution of $\ln \Omega(V)=\ln (\mathcal{V})-\ln (\langle v\rangle)$ and of Eq. (10) for $\mathcal{U}(x \mid V)$, we write an expression for the 

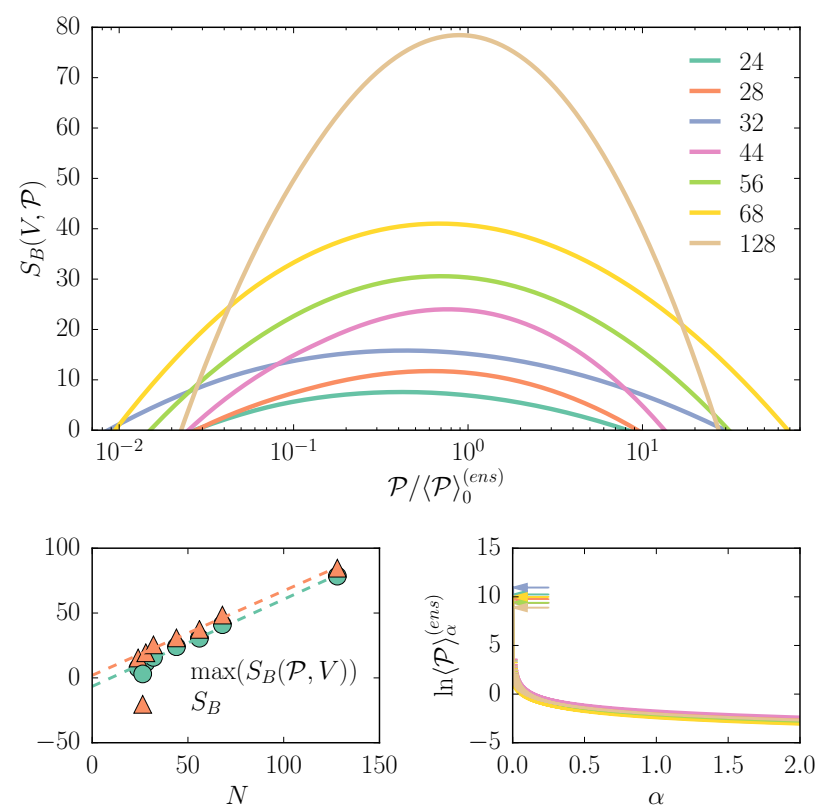

FIG. 6: Top: generalised Edwards entropy at fixed volume fraction for various system sizes. The curves show a well defined maximum for all sizes, while their shape depends on the specific parameters of the generalised log-normal that best fits the underlying distribution of pressures. Bottom left: comparison between the Edwards entropy and the maximum value attained by each curve: $\max \left[S_{B}(\mathcal{P}, V)\right]$ scales linearly with size and its value is progressively closer to the marginal (total) Edwards entropy $S_{B}(V)$, consistent with the fact that $S_{B}(\mathcal{P}, V)$ is a negative exponential function, and the area under the curve is dominated by the mode for increasing system size. $S_{B}(V)$ should constitute an upper limit to $\max \left[S_{B}(\mathcal{P}, V)\right] \leq S_{B}(V)$ and the two should be equivalent only in the thermodynamic limit. Bottom right: ensemble average of the pressure computed as a function of inverse angoricity $\alpha$ and system size. The curves, in the same colour as the top figure, do not diverge and the arrows indicates their value at $\alpha=0$.

Edwards entropy as a function of volume and pressure

$$
\begin{aligned}
S_{B}(V, \mathcal{P})= & \ln \left(\int_{\mathcal{P}}^{\mathcal{P}+\delta \mathcal{P}} \mathcal{B}(x \mid V) x^{N / \kappa} \mathrm{d} x\right) \\
& +\ln (\mathcal{V})+\mathcal{C}(N)-\ln (N !) .
\end{aligned}
$$

We fit the empirical cumulative distribution function (c.d.f.) of $\mathcal{B}(\mathcal{P})$ with the generalised log-normal c.d.f. corresponding to Eq. (28) (see Fig. 5). We then numerically evaluate the generalised Edwards entropy $S_{B}(\mathcal{P}, V)$ at fixed volume, as shown in Fig. 6.

In the thermodynamic limit we find

$$
s_{B}\left(\phi_{\mathrm{SS}}\right)=1+c+\frac{\langle\ln (\mathcal{P})\rangle_{\mathcal{B}}}{\kappa}-\ln \left(\phi_{\mathrm{SS}}\right)-f_{\mathrm{ex}}\left(\phi_{\mathrm{HS}}\right),
$$

where $c=\mathcal{C}(N) / N$, see Appendix F for details of the derivation and further discussion.

In Fig. 6 we also show the predicted expectation value for the pressure obtained via the ensemble average at arbitrary inverse angoricity $\alpha$,

$$
\langle\mathcal{P}\rangle_{\alpha}^{(\text {ens })}=\frac{\int_{0}^{\infty} \mathcal{P} \mathcal{B}(\mathcal{P} \mid V) \mathcal{P}^{N / \kappa} e^{-\alpha \mathcal{P} V} \mathrm{~d} \mathcal{P}}{\int_{0}^{\infty} \mathcal{B}(\mathcal{P} \mid V) \mathcal{P}^{N / \kappa} e^{-\alpha \mathcal{P} V} \mathrm{~d} \mathcal{P}} .
$$

\section{PACKING PREPARATION PROTOCOL}

\section{A. Sampling packings}

The physical properties of granular packings may depend strongly on the preparation protocol. This is illustrated by the Lubachevsky-Stillinger algorithm (LSA) procedure to prepare jammed packings of hard particles [90] by compression (or, equivalently, by 'inflation' of the particles). If a monodisperse HS fluid is compressed rapidly the LSA will generate a low volume-fraction disordered packing. However, for (very) slow compression rates, LSA will produce dense crystals [90, 91].

In the present work, we study a fluid of polydisperse spheres. We use a protocol related to a Stillinger-Weber quench that maps each fluid state to a local minimum, or "inherent structure", connected by a path of steepest descent [86, 92].

To prepare the polydisperse fluid, we draw $N$ particle radii $\{r\}_{N}$ from a Gaussian distribution $\operatorname{Normal}\left(1, \sigma_{\mathrm{HS}}\right)>0$, truncated at $r=0$ (note that in our application the standard deviation $\sigma_{\mathrm{HS}}$ is sufficiently small that it is extremely improbable to ever sample a negative radius). We set the box size to meet the target packing fraction of the hard sphere fluid $\phi_{\mathrm{HS}}$ and then place the particles in a random valid initial hard spheres configuration. The initial configuration is then evolved by a MC simulation [93] consisting of single particle random displacements and particle-particle swaps, and after equilibration, new configurations are recorded at regular intervals. We choose the length of these intervals such that, on average, each particle diffuses over a distance equal to the diameter of the largest particle. As long as $\phi_{\text {HS }}$ is well below the volume fraction where the fluid undergoes structural arrest, the allowed configurations of the fluid can be sampled uniformly. Importantly, this volume fraction is well below the random close packing $\left(\phi_{\mathrm{HS}}^{(\mathrm{RCP}, 3 \mathrm{D})} \approx 0.64\right.$ and $\left.\phi_{\mathrm{HS}}^{(\mathrm{RCP}, 2 \mathrm{D})} \approx 0.82[79]\right)$.

Given these HS fluid configurations, we now switch on the soft, repulsive interaction to generate overcompressed jammed packings of the particles (see Fig. 2). The particles are inflated with a WCA-like potential [94] to reach the target soft packing fraction $\phi_{\mathrm{SS}}>\phi_{\mathrm{HS}}^{(\mathrm{RCP})}>$ $\phi_{\text {HS }}$. The hard spheres are inflated proportional to their 
radius, so that the soft sphere radius is

$$
r_{s}=\left(\frac{\phi_{\mathrm{SS}}}{\phi_{\mathrm{HS}}}\right)^{1 / d} r_{h}
$$

where $d$ is the dimensionality of the box. Clearly, this procedure does not change the polydispersity of the sample.

\section{B. Soft shells and minimisation}

We define the WCA-like potential around a hard core as follows: consider two spherical particles with hard core distance $r_{h}$ and soft core contact distance $r_{s}=r_{h}(1+$ $\theta)$, with $\theta=\left(\phi_{\mathrm{SS}} / \phi_{\mathrm{HS}}\right)^{1 / d}-1$. We can then write a horizontally shifted hard-sphere plus WCA (HS-WCA) potential as

$$
v_{\mathrm{HS}-\mathrm{WCA}}(r)= \begin{cases}\infty & r \leq r_{h}, \\ 4 \epsilon\left[\left(\frac{\sigma\left(r_{h}\right)}{r^{2}-r_{h}^{2}}\right)^{12}\right. & \\ \left.-\left(\frac{\sigma\left(r_{h}\right)}{r^{2}-r_{h}^{2}}\right)^{6}\right]+\epsilon & \\ 0 & r \geq r<r_{s}\end{cases}
$$

where $\sigma\left(r_{h}\right)=\left(2 \theta+\theta^{2}\right) r_{h}^{2} / 2^{1 / 6}$ guarantees that the potential goes to zero at $r_{s}$. For computational convenience (avoidance of square-root evaluations), the potential in Eq. 15 differs from the WCA form in that the interparticle distance in the denominator of the WCA potential has been replaced with a difference of squares. Note that this implies that our potential resembles a 6-3 potential more than a 12-6 potential. For our purpose, this difference is immaterial: we just need a short-ranged repulsive potential that diverges at the hard-core diameter and vanishes continuously at the soft-core diameter. The functional form of this potential is very similar to the HS-WCA potential used by Asenjo et al. [34], but cheaper to compute. We note that this potential is a $C^{1}$ type function, that is, its first derivative is continuous but not differentiable and its second derivative is discontinuous at $r_{s}$. We take advantage of this property for the identification of rattlers (non-jammed particles) in our packings.

Numerically evaluating this potential, we match the gradient and linearly continue the function $v_{\mathrm{HS}-\mathrm{WCA}}(r)$ for $r \leq r_{h}+\varepsilon$, with $\varepsilon>0$ an arbitrary small constant, such that minimisation is still meaningful if overlaps do occur.

The HS-WCA pair-potential was implemented using cell-lists $[95,96]$ with periodic boundary conditions, guaranteeing $\mathcal{O}(N)$ time complexity to the energy and gradient evaluations. Energy minimisations were performed with the CG_DESCENT algorithm [97-99] which, compared to FIRE [96, 100], reduces the average number of function evaluations for our system by a factor of 5 , while preserving many of its desirable properties.

\section{BASIN VOLUME BY THERMODYNAMIC INTEGRATION}

The basin of attraction of a given minimum-energy configuration is the collection of all points connected to that minimum via a path of steepest descent $[81,101]$. To measure the volume of a basin of attraction in the PES, we use thermodynamic integration $[102,103]$ and parallel tempering (PT) [93, 104-106].

The basic idea behind the method is that, but for the sign, the logarithm of the basin volume can be viewed as a dimensionless free energy. We cannot determine this free energy directly. We now switch on an increasingly harmonic potential that has its minimum at the minimum of the basin. In the limit of very large coupling constants (how large depends on the shape of the basin) the boundaries of the basin no longer affect the free energy of the system, which has effectively been reduced to a $d N$ dimensional harmonic oscillator with known free energy (for more details, see Appendix D). For zero coupling constant, instead, the system is completely unconstrained and therefore in the state of interest. Thermodynamic integration allows us to compute the free energy difference between a reference state of known free energy and the (unknown) free energy associated with the original basin of attraction.

A closely related approach is often used to compute the free energy of crystals of particles with a discontinuous potential, such as hard spheres [102, 103, 107]. Details of that method are summarised in the Appendix B, and the extension of the technique to basin volume measurement is described below. Details of the Hamiltonian PT are discussed in Appendix C.

\section{A. Free energy calculation for basin volumes}

To measure the volume of a basin by thermodynamic integration, we perform a walk inside the basin, that is, we start the MCMC random walk from the minimum energy configuration $\mathbf{r}_{i}$ and we reject every move that takes us outside the basin $[34,36,83]$. This procedure can be cast in normal Monte Carlo language by defining an effective potential energy function (oracle) $U_{B}\left(\mathbf{r} \mid \mathbf{r}_{i}\right)$ which is zero inside the basin and infinite outside. We can then write the volume of the basin:

$$
v_{i}=\int \mathrm{d} \mathbf{r} e^{-U_{B}\left(\mathbf{r} \mid \mathbf{r}_{i}\right)}
$$

In order for the oracle to test whether a proposed configuration is inside or outside the basin, a full energy minimisation must be performed. The numerous potential energy calls required for a full energy minimisation represent the major obstacle to the scalability of the method.

We view the negative log-basin-volume as a dimensionless free energy $F_{i} \equiv-\ln \left(v_{i}\right)$ [36] and compute it by thermodynamic integration, as described in Appendix B. 
Therefore, we write, analogously to Eq. (B2):

$$
-\ln v_{i}=F_{\text {har }}\left(k_{\max }\right)-\frac{1}{2} \int_{0}^{k_{\max }} \mathrm{d} k\left\langle\left|\mathbf{r}-\mathbf{r}_{i}\right|\right\rangle_{k},
$$

where $\mathbf{r}_{i}$ denotes the coordinates of the $i$-th energy minimum. Unless $k_{\max }$, the maximum spring constant of the harmonic reference system, is very large, a finite fraction of the points belonging to the purely harmonic reference system will be located in the region where $U_{B}=\infty$.

We can correct for this effect in our calculation of $F_{\text {har }}\left(k_{\max }\right)$ by computing the ratio of the partition functions of a system with a harmonic spring constant $k_{\max }$, both with and without the basin potential energy function $U_{B}$. This ratio is given by

$$
\mathcal{R} \equiv \frac{\int \mathrm{d} \mathbf{r} \exp \left[-V\left(\mathbf{r} \mid \mathbf{r}_{i}, k_{\max }\right)-U_{B}\left(\mathbf{r} \mid \mathbf{r}_{i}\right)\right]}{\int \mathrm{d} \mathbf{r} \exp \left[-V\left(\mathbf{r} \mid \mathbf{r}_{i}, k_{\max }\right)\right]},
$$

where $V$ is the sum of the hard-core potential and the harmonic potential with spring constant $k_{\max }$, see Eq. (B1). We note that $\mathcal{R}$ can be computed using a 'static' (i.e. non-Markov chain) Monte Carlo simulation, sampling directly from the Boltzmann distribution of the harmonic oscillator with spring constant $k_{\max }$. Since the integral in the denominator is known [see Eq. (D2)], we write the dimensionless free energy of the harmonic reference state for basin $i$ as

$$
F_{\text {har }}\left(k_{\max }\right)=-\frac{d N}{2} \ln \left(\frac{2 \pi}{k_{\max }}\right)-\ln \mathcal{R} .
$$

We note that, in order to avoid a singularity in the integrand, it is useful to perform the simulations fixing the centre of mass. It follows that the same corrections to the free energy as derived in Refs.[102, 103, 107] must be applied: similarly to Eq. (B5), but with the additional correction in Eq. (19), we write the basin volume as:

$$
\begin{aligned}
-\ln v_{i} & =\Delta F^{(\mathrm{CM})}-\ln \left(V_{\mathrm{box}}\right) \\
- & \frac{(N-1) d}{2} \ln \left(\frac{2 \pi}{k_{\max }}\right)-\ln \mathcal{R},
\end{aligned}
$$

where $\Delta F^{(\mathrm{CM})}$ is the integral in Eq. (17), and the ensemble averages have been computed with a constrained centre of mass and it is evaluated as in Eq. (B13).

Figure 7 shows an example of the mean squared displacement $\left\langle\left|\mathbf{r}-\mathbf{r}_{0}\right|^{2}\right\rangle_{k}$, as a function of the spring constant $k$, along with the approximate expression in Eq. (B8) used to construct the change of variables in Eq. (B13). The resulting integrand, after the variable transform, is shown in the inset of Fig. 7.

\section{BASIN VOLUME DISTRIBUTIONS AND DATA ANALYSIS}

Once the volumes of multiple basins have been sampled, these data can be used to compute the number of

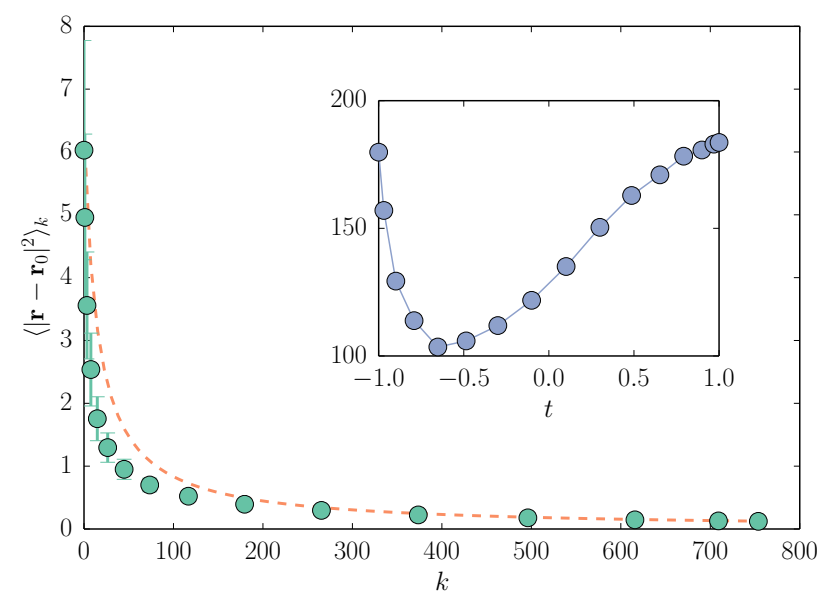

FIG. 7: Average squared displacement $\left\langle\left|\mathbf{r}-\mathbf{r}_{0}\right|^{2}\right\rangle_{k}$ as a function of the spring constant $k$ (symbols). The dashed line shows the expression in Eq. (B8). The data is measured for a packing of $N=32$ spheres, with $\phi_{\mathrm{HS}}=0.5$ and $\phi_{\mathrm{SS}}=0.7$ via Hamiltonian PT. Inset: corresponding integrand for the thermodynamic integration, resulting from the change of variables in Eq. (B13).

distinct packings [34], and from that, the Edwards entropy [4]. Furthermore we analyse the distribution of pressures of the different energy minima at given volume. In this work, we express pressure and volume in reduced units $\mathcal{P} / \mathcal{P}^{*}$ and $v / v^{*}$ everywhere with $v^{*} \equiv(4 \pi / 3)\left\langle r_{h}^{3}\right\rangle$ and $\mathcal{P}^{*} \equiv \epsilon / v^{*}$ being the units of volume and pressure, respectively.

\section{A. Gibbs configurational entropy}

Let us first consider the 'Gibbs' configurational entropy, $S_{G}$, defined by Asenjo et al. [34]:

$$
S_{G}=-\sum_{i=1}^{\Omega} p_{i} \ln \left(p_{i}\right)-\ln (N !),
$$

where $p_{i}$ is the probability to sample packing $i$. For our preparation protocol, packings are sampled according to the volume of their basin of attraction, such that $p_{i}=$ $v_{i} / \mathcal{V}$. Then Eq. (21) gives

$$
\begin{aligned}
S_{G} & =-\sum_{i=1}^{\Omega} p_{i} \ln \left(v_{i}\right)+\ln (\mathcal{V})-\ln (N !) \\
& =\langle F\rangle_{\mathcal{B}}+\ln (\mathcal{V})-\ln (N !) .
\end{aligned}
$$

The sum in Eq. (22) is the mean of the negative logbasin volumes (dimensionless free energies), as computed above, and weighted by the probabilities of preparing the corresponding basins. Therefore, the entropy can be obtained directly, and without approximation, from the sampled mean basin dimensionless free energy. 
From Eq. (22) we can also write the entropy per particle in the thermodynamic limit as

$$
s_{G}\left(\phi_{\mathrm{SS}}\right)=1+\langle f\rangle_{\mathcal{B}}+\ln \left(\phi_{\mathrm{SS}}\right)-f_{\mathrm{ex}}\left(\phi_{\mathrm{HS}}\right),
$$

where $f_{\mathrm{ex}}\left(\phi_{\mathrm{HS}}\right)$ is the excess free energy of the hard spheres fluid. In deriving this results we used Stirling's approximation for large $N$ and the fact that $V_{\mathrm{box}} / v^{*}=$ $N / \phi_{\mathrm{SS}}$.

\section{B. Edwards configurational entropy}

Edwards [4] suggested a Boltzmann-like entropy, where $S$ equals the logarithm of $\Omega$, the total number of packings. Asenjo et al. [34] showed that, even for polydisperse particles, indistinguishability of macrostates requires that

$$
S_{B}=\ln (\Omega)-\ln (N !),
$$

The subtraction of $\ln (N$ !) is necessary to guarantee extensivity of the entropy. Unlike the Gibbs definition of entropy, Eq. (24) makes the explicit assumption of equiprobability of states.

For a direct computation of the number of packings $\Omega$, using Eq. (3), we need the average basin volume $\langle v\rangle$. Since our preparation protocol samples each minimum with a probability proportional to the volume of its basin of attraction, our samples of $v$ are biased accordingly. Therefore, to obtain the unbiased average basin volume $\langle v\rangle$, the sampled basin volume distribution needs to be unbiased [34, 35, 83]. The unbiasing method used in the following work requires an analytical (or at least numerically integrable) description of the biased basin free energy distribution function. Different approaches to modelling this distribution give rise to somewhat different analysis methods, which all yield consistent results. Again, we stress that no such additional steps are needed to compute the 'Gibbs' version of the configurational entropy.

We distinguish between the biased, $\mathcal{B}\left(F \mid N, \phi_{\mathrm{SS}}\right.$ ) (as sampled by the packing protocol), and the unbiased, $\mathcal{U}\left(F \mid N, \phi_{\mathrm{SS}}\right)$, free energy distributions. Since the configurations were sampled proportional to the volume of their basin of attraction, we can compute the unbiased distribution as

$$
\mathcal{U}\left(F \mid N, \phi_{\mathrm{SS}}\right)=\mathcal{Q}\left(N, \phi_{\mathrm{SS}}\right) \mathcal{B}\left(F \mid N, \phi_{\mathrm{SS}}\right) e^{F}
$$

where $\mathcal{Q}\left(N, \phi_{\mathrm{SS}}\right)$ is the normalisation constant

$\mathcal{Q}\left(N, \phi_{\mathrm{SS}}\right)=\left[\int_{F_{\min }}^{\infty} \mathrm{d} F \mathcal{B}\left(F \mid N, \phi_{\mathrm{SS}}\right) e^{F}\right]^{-1}=\langle v\rangle\left(N, \phi_{\mathrm{SS}}\right)$.

From Eq. (25), unbiasing of the raw free energy distribution seems straightforward, however Asenjo at al. [34] noted that the most probable basins are about $O\left(10^{3}\right)$ more probable than the small ones. Upon unbiasing, this factor is multiplied by a factor of about $e^{-20}$, hence they observe that small basins are much more numerous than large ones and grossly under-sampled.

To overcome this problem, one can fit the biased measured free energy distribution $\mathcal{B}\left(F \mid N, \phi_{\mathrm{SS}}\right)$ and perform the unbiasing via Eq. (26) on the best fitting distribution. $\mathcal{B}\left(F \mid N, \phi_{\mathrm{SS}}\right)$ must be bounded, hence it should decay with a functional form $\exp \left(-F^{\nu}\right)$ where $\nu>1$.

Before performing the fit we remove outliers from the free energy distribution following the distance-based outlier removal method introduced by Knorr and $\mathrm{Ng}$ [108]. This is a form of clustering for which we choose to keep only those points for which at least half of the remaining data set is within $3 \sigma$ from the point, where $\sigma$ is the standard deviation computed for the raw data set. This procedure typically results in the exclusion of one or two points and it is essential for a successful fit to a generalised Gaussian model.

\section{Generalised Gaussian}

Assuming that $\mathcal{U}\left(F \mid N, \phi_{\mathrm{SS}}\right)$ is unimodal, which has been verified for very small systems [36], one can fit the raw distribution $\mathcal{B}\left(F \mid N, \phi_{\mathrm{SS}}\right)$ with a three-parameter generalised normal distribution

$$
p(F \mid \bar{F}, \sigma, \zeta) \equiv \frac{\zeta}{2 \sigma \Gamma(1 / \zeta)} \exp \left[-\left(\frac{|F-\bar{F}|}{\sigma}\right)^{\zeta}\right],
$$

where $\Gamma(x)$ is the gamma function, $\sigma$ is the scale parameter, $\zeta$ is the shape parameter and $\bar{F}$ is the mean (free energy) with variance $\sigma^{2} \Gamma(3 / \zeta) / \Gamma(1 / \zeta)$. In the limit $\zeta \rightarrow 2$ we recover the Gaussian distribution with standard deviation $\sigma$. In practice it appears to be most stable to fit the empirical biased cumulative distribution function, rather than the histogram shape [34]. Alternatively, we also tested fitting to the observed p.d.f. with the maximumlikelihood method, obtaining consistent, but more scattered, results (see also Sec. III).

\section{Kernel density estimate}

To relax the assumption that the empirical distributions can be fitted by a generalised Gaussian, one can also describe the distributions by kernel density estimation [109, 110]. Bandwidth selection is then done using Silverman's rule of thumb as the initial guess for integrated squared error cross-validation [111]. The numerical integration step is performed, as for the generalised Gaussian description, via Eq. (26).

\section{Distribution of pressures}

In Sec. III B we have established a link between the pressure of a packing and the volume of its basin of at- 
traction. In order to compute the entropy as a function of volume and pressure it is necessary to unbias the distribution of pressures with respect to the sampling bias $\exp (-F)$, analogous to the previous section. We choose to describe the distribution of pressures $\mathcal{P}$ using the generalised log-normal distribution [112]

$$
\begin{aligned}
p(\mathcal{P} \mid \overline{\ln (\mathcal{P})}, \sigma, \zeta)= & \frac{\zeta / \mathcal{P}}{2^{(\zeta+1) / \zeta} \sigma \Gamma(1 / \zeta)} \\
& \exp \left(-\frac{1}{2}\left|\frac{\ln (\mathcal{P})-\overline{\ln (\mathcal{P})}}{\sigma}\right|^{\zeta}\right),
\end{aligned}
$$

with the first term on the r.h.s. being the normalisation constant and the remaining notation analogous to that of Eq. (27). For $\zeta=2$ this distribution reduces to the log-normal distribution.

\section{CONCLUSIONS}

The study of a statistical mechanics of granular materials has been complicated by the impossibility of directly computing fundamental thermodynamic quantities. In the present paper we have shown that configurational entropies of three-dimensional packings can, in fact, be computed.

We have presented a method for the direct enumeration of the mechanically stable states of systems consisting of up to 128 frictionless soft three-dimensional spheres and we have shown that a definition of extensive entropy is possible, in line with the results for two dimensional systems reported by Asenjo et al. [34], with very minor differences in our observations. The study of 3D packings is computationally demanding: the computational time required for each packing ranged between 10 and $10^{4} \mathrm{cpu}$ hours, depending on system size. The present study therefore required substantial algorithmic optimisation.

We find that there is an approximately linear relationship between the logarithm of the pressure of a mechanically stable configuration and the logarithm of the volume of its basin of attraction.

The unexpected power law relationship between pressure and basin volume provides a way to extend our approach to the generalised Edwards ensemble. We can analytically unbias the observed distribution of pressures and compute the entropy as a function of pressure at a given volume. Hence we have obtained consistent expressions for the entropy in the thermodynamic limit. Knowledge of this distribution enables the first direct computation of angoricity.

Tackling the study of granular materials from the energy landscapes point of view is rather advantageous, although this does not come without burdens. This sort of approach is limited to soft frictionless particles, and we expect it to be reliable only at $\phi>\phi_{J}$ when the system is at least slightly over-compressed. Other theoretical approaches are useful in more limiting situations, see for instance the discussion of the stress ensemble in the limit $\phi \rightarrow \phi_{J}$ by Henkes and Chakraborty [26, 27] and the work on the force network ensemble for systems of almost hard grains [31-33].

\section{ACKNOWLEDGMENTS}

We acknowledge useful discussions with Daniel Asenjo, Carl Goodrich, Silke Henkes, and Fabien Paillusson. S.M. acknowledges financial support by the Gates Cambridge Scholarship. K.J.S. acknowledges support by the Swiss National Science Foundation under Grant No. P2EZP2-152188 and No. P300P2-161078. J.D.S. acknowledges support by Marie Curie Grant 275544. D.F. and D.J.W. acknowledge support by EPSRC Programme Grant EP/I001352/1, by EPSRC grant EP/I000844/1 (D.F.) and ERC Advanced Grant RG59508 (D.J.W.)

\section{Appendix A: Basin volume method summary}

In summary, to count the number of ways spheres can pack into a given volume, we use the mean basin volume method outlined in Sec. II. We perform the following simulations and analysis steps to obtain the required results:

1. Obtain a number of different snapshots of an equilibrated hard sphere fluid at the desired volume fraction $\phi_{\mathrm{HS}}$, as described in Sec. IV A. This procedure fixes the number of measured basin volumes.

2. Over-compress the sphere configuration by adding a soft shell. This compression yields, upon energy minimisation, a jammed packing with soft volume fraction $\phi_{\mathrm{SS}}>\phi_{\mathrm{HS}}$.

3. Estimate the maximum spring constant for the PT simulations, $k_{\max }$ in Eq. (17), such that $\rho$ in Eq. (18) reaches a value between 0.85 and 0.9 . This is done by direct sampling and also gives the value of the average squared displacement for $k_{\max }$, $\left\langle\left|\mathbf{r}-\mathbf{r}_{0}\right|^{2}\right\rangle_{k_{\max }}$.

4. Obtain a preliminary estimate of the average squared displacement without harmonic tethering, $\left\langle\left|\mathbf{r}-\mathbf{r}_{0}\right|^{2}\right\rangle_{0}$, by performing a MCMC walk in the basin. Use this result, with the estimate of $k_{\max }$ from the previous step, to determine the spring constants $k$ for the PT simulation, using Eqs. (B11) and (B14).

5. Perform a PT simulation to sample $\left\langle\left|\mathbf{r}-\mathbf{r}_{0}\right|^{2}\right\rangle_{k}$, as described in Sec. C.

6. Compute the volume in Eq. (20) for each basin and analyse the distributions for all basins, at fixed volume fraction and number of particles, as discussed in Sec. VI. This makes use of the total accessible volume, computed in Appendix E. 
Section III shows examples of the type of results that can be obtained. Evaluation and minimisation of potential energy functions was performed with the pele [96] and PyCG_DESCENT [99] software packages. Monte Carlo simulations were performed with the mcpele package [93].

\section{Appendix B: Free energy calculation for solids}

To compute the free energy of a system with discontinuous potential energy function (e.g., hard disks or hard spheres), we construct a reversible path to the corresponding Einstein solid (see e.g. [107]). The harmonic potential with spring constant $k$ is switched on while maintaining the hard core interactions intact:

$$
\begin{aligned}
V\left(\mathbf{r} \mid \mathbf{r}_{0}, k\right) & =V_{\mathrm{HS}}(\mathbf{r})+k V_{\mathrm{har}}\left(\mathbf{r} \mid \mathbf{r}_{0}\right) \\
& =V_{\mathrm{HS}}(\mathbf{r})+\frac{1}{2} k\left|\mathbf{r}-\mathbf{r}_{0}\right|^{2}
\end{aligned}
$$

where $\mathbf{r}_{0}$ are the equilibrium coordinates of the Einstein crystal and $V_{\mathrm{HS}}(\mathbf{r})$ denotes the hard core interactions. We can then compute the free energy difference between the Einstein crystal and the hard core system by evaluating the integral:

$$
F_{\mathrm{HS}}=F_{\text {har }}\left(k_{\max }\right)-\int_{0}^{k_{\max }} \mathrm{d} k\left\langle\frac{\partial V\left(\mathbf{r} \mid \mathbf{r}_{0}, k\right)}{\partial k}\right\rangle_{k} .
$$

As discussed in Appendix D, we take the centre of mass to be fixed to avoid numerical issues in the limit $k \rightarrow 0$. For a system with fixed centre of mass, we write the free energy difference between the target and the reference state as

$$
\Delta F^{(\mathrm{CM})} \equiv F^{(\mathrm{CM})}-F_{\mathrm{har}}^{(\mathrm{CM})} .
$$

From the partition function of the Einstein crystal with fixed centre of mass, Eq. (D7), and for the unconstrained crystal, Eq. (D11), we can rewrite Eq. (B3) and rearrange it for the free energy of the unconstrained crystal:

$$
\begin{aligned}
F & =\Delta F^{(\mathrm{CM})}+\ln \left(\mathcal{P}\left(\mathbf{r}_{\mathrm{CM}}=\mathbf{0}\right)\right) \\
& +\frac{d}{2} \ln \left(\frac{2 \pi \sum_{i} \mu_{i}}{k_{\max }}\right)-\frac{N d}{2} \ln \left(\frac{2 \pi}{k_{\max }}\right),
\end{aligned}
$$

where the last term is $F_{\text {har }}$ and the second and third terms on the right hand side are the CM corrections for the unconstrained and the constrained solid, respectively. For a system with unit cell identical to the simulation box (with periodic boundary conditions), we have $\mathcal{P}\left(\mathbf{r}_{\mathrm{CM}}=\right.$ $\mathbf{0})=1 / V_{\text {box }}$. Assuming that all particles have unit mass we can rewrite Eq. (B4) as

$$
F=\Delta F^{(\mathrm{CM})}-\ln \left(V_{\mathrm{box}}\right)-\frac{(N-1) d}{2} \ln \left(\frac{2 \pi}{k_{\max }}\right) .
$$

We are only left with $\Delta F^{(\mathrm{CM})}$, which can be found by evaluating the integral in Eq. (B2). In order to do so, we would like the integrand to be a well behaved function, possibly flat, permitting Gauss-Lobatto (GL) quadrature [113]. We transform the integration variable so that

$$
\begin{aligned}
\Delta F^{(\mathrm{CM})} & =\int_{0}^{k_{\max }} \frac{\mathrm{d} k}{g(k)} g(k) \frac{1}{2}\left\langle\left|\mathbf{r}-\mathbf{r}_{0}\right|^{2}\right\rangle_{k}^{(\mathrm{CM})} \\
& =\int_{G^{-1}(0)}^{G^{-1}\left(k_{\max }\right)} \mathrm{d}\left[G^{-1}(k)\right] g(k) \frac{1}{2}\left\langle\left|\mathbf{r}-\mathbf{r}_{0}\right|^{2}\right\rangle_{k}^{(\mathrm{CM})},
\end{aligned}
$$

where $g(k)$ is some function of $k$ and $G^{-1}(k)$ is the primitive of the function $1 / g(k)$.

To choose an appropriate $g(k)$, we note that in Eq. (D8) for very large $k$ the mean squared displacement for the solid is

$$
\left\langle\left|\mathbf{r}-\mathbf{r}_{0}\right|^{2}\right\rangle_{k_{\max }}=\frac{(N-1) d}{k_{\max }} .
$$

For $k$ other than $k_{\max }$, we expect the mean squared displacement to depend on some effective spring constant. Hence we write

$$
\left\langle\left|\mathbf{r}-\mathbf{r}_{0}\right|^{2}\right\rangle_{k} \approx \frac{(N-1) d}{(k+\xi)},
$$

such that the mean squared displacement at $k=0$ is

$$
\left\langle\left|\mathbf{r}-\mathbf{r}_{0}\right|^{2}\right\rangle_{k=0} \approx \frac{(N-1) d}{\xi},
$$

from which we find $\xi=(N-1) d /\left\langle\left|\mathbf{r}-\mathbf{r}_{0}\right|^{2}\right\rangle_{k=0}$ [note that we can self consistently replace this definition for $\xi$ in Eq. (B8) to obtain an approximation for the mean squared displacement at arbitrary $k$. We would like the integrand $g(k)\left\langle\left|\mathbf{r}-\mathbf{r}_{0}\right|^{2}\right\rangle_{k}$ in Eq. (B6) to be roughly constant. Given the considerations above we choose $g(k) \approx k+\xi$. One can easily verify that the integrand is now approximately constant. We can then rewrite the integral in Eq. (B6) as

$$
\begin{array}{r}
\Delta F^{(\mathrm{CM})}=\int_{\ln (\xi)}^{\ln \left(k_{\max }+\xi\right)}\left\{(k+\xi) \frac{1}{2}\left\langle\left|\mathbf{r}-\mathbf{r}_{0}\right|^{2}\right\rangle_{k}^{(\mathrm{CM})}\right. \\
\mathrm{d}[\ln (k+\xi)]\} .
\end{array}
$$

Finally, to integrate Eq. (B10) by GL quadrature, we require a variable, $t$, such that the integral upper and lower bounds are $[-1,1]$ :

$$
t=\frac{2 \ln (1+k / \xi)-1}{\ln \left(1+k_{\max } / \xi\right)}
$$

with differential

$$
\mathrm{d} t=\frac{2}{\ln \left(1+k_{\max } / \xi\right)} \mathrm{d}[\ln (1+k / \xi)] .
$$

Therefore we rewrite Eq. (B10) as a function of $t$ :

$$
\begin{aligned}
& \Delta F^{(\mathrm{CM})}=\int_{-1}^{1}\left\{\mathrm{~d} t \ln \left(1+\frac{k_{\max }}{\xi}\right)\right. \\
& {\left.[k(t)+\xi] \frac{1}{4}\left\langle\left|\mathbf{r}-\mathbf{r}_{0}\right|^{2}\right\rangle_{k}^{(\mathrm{CM})}\right\}, }
\end{aligned}
$$


where $k(t)$ can be found by rearranging Eq. (B11). An example of the variable transform is shown in Fig. 7.

It is straightforward to perform GL quadrature for a general number of abscissas $n \geq 2$ [113], because

$$
\int_{-1}^{1} \mathrm{~d} t f(t)=w_{1} f(-1)+\sum_{i=2}^{n-1} w_{i} f\left(t_{i}\right)+w_{n} f(1),
$$

where $w_{i}$ are the weights and $t_{i}$ are the abscissas. The abscissas different from $-1,1$ are the $n-2$ roots of $\mathrm{d} P_{n-1}(t) / \mathrm{d} t$, with $P_{n-1}$ a Legendre polynomial. We evaluate this sum numerically using Numpy's Legendre module [114]. The weights $w_{i}$ can also be evaluated numerically for general $n \geq 2$, since they are related to $P_{n-1}$ evaluated at $t_{i}$ [113]. For all results in this work, we choose $n=16$ abscissas.

\section{Appendix C: Sampling the integrand: Hamiltonian Parallel Tempering}

To compute the integral in Eq. (B13), we need to measure the integrand for different values of $k$, as given by Eq. (B11). Equilibration of the corresponding simulations can be accelerated using extensions of the parallel tempering technique, where replicas differ in chemical potential [115] or in the potential energy function [116, 117].

The Parallel-tempering acceptance rule for a swap of configurations between replicas with different Hamiltonians follows from the condition of detailed balance:

$$
\begin{aligned}
& \frac{\operatorname{acc}\left[\left(\mathbf{r}_{i}, V_{i}\right),\left(\mathbf{r}_{j}, V_{j}\right) \rightarrow\left(\mathbf{r}_{j}, V_{i}\right),\left(\mathbf{r}_{i}, V_{j}\right)\right]}{\operatorname{acc}\left[\left(\mathbf{r}_{i}, V_{j}\right),\left(\mathbf{r}_{j}, V_{i}\right) \rightarrow\left(\mathbf{r}_{i}, V_{i}\right),\left(\mathbf{r}_{j}, V_{j}\right)\right]} \\
& =\frac{\exp \left\{-\beta\left[V_{i}\left(\mathbf{r}_{j}\right)+V_{j}\left(\mathbf{r}_{i}\right)\right]\right\}}{\exp \left\{-\beta\left[V_{i}\left(\mathbf{r}_{i}\right)+V_{j}\left(\mathbf{r}_{j}\right)\right]\right\}} \\
& =\exp \left\{-\beta\left[\left(V_{i}\left(\mathbf{r}_{j}\right)+V_{j}\left(\mathbf{r}_{i}\right)\right)-\left(V_{i}\left(\mathbf{r}_{i}\right)+V_{j}\left(\mathbf{r}_{j}\right)\right)\right]\right\},
\end{aligned}
$$

where acc $[\cdot \rightarrow \cdot]$ denotes the swap acceptance probability. For the particular case of replicas coupled to a reference state $\mathbf{r}_{0}$ by a harmonic potential with different coupling strengths $k_{i}$, we find the swap acceptance rule

$$
\begin{aligned}
& \operatorname{acc}\left[\left(\mathbf{r}_{i}, V_{i}\right),\left(\mathbf{r}_{j}, V_{j}\right) \rightarrow\left(\mathbf{r}_{j}, V_{i}\right),\left(\mathbf{r}_{i}, V_{j}\right)\right] \\
& =\min \left\{1, \exp \left[\frac{\beta}{2}\left[\left(k_{j}-k_{i}\right)\left(\left|\mathbf{r}_{j}-\mathbf{r}_{0}\right|^{2}-\left|\mathbf{r}_{i}-\mathbf{r}_{0}\right|^{2}\right)\right]\right\} .\right.
\end{aligned}
$$

To check whether the replicas are well equilibrated, we consider the correlations in the "time series" of $\left|\mathbf{r}-\mathbf{r}_{0}\right|_{k}$ vs number of Monte Carlo steps for each replica.

\section{Appendix D: Einstein crystal}

The harmonic potential is defined as follows:

$$
V\left(\mathbf{r} \mid \mathbf{r}_{0}, k\right)=\frac{k}{2}\left|\mathbf{r}-\mathbf{r}_{0}\right|^{2}=\frac{k}{2} \sum_{i}^{N}\left|\mathbf{r}_{i}-\mathbf{r}_{i, 0}\right|^{2}
$$

where $\mathbf{r}_{0}$ denotes the equilibrium position, the index $i$ denotes the $i$-th of $N$ particles, each with $d$ degrees of freedom, and we have assumed that the spring constant $k$ is the same for all directions of motion. We can compute the mean squared particle displacement for a harmonic oscillator in the canonical ensemble analytically. We start with the partition function:

$$
Z_{N V T}=\left(\frac{2 \pi}{\beta k}\right)^{\frac{d N}{2}}
$$

We consider the free energy for the system $F=$ $-\beta^{-1} \ln Z$ and observe that

$$
\begin{aligned}
\left(\frac{\partial F(k)}{\partial k}\right)_{N V T} & =-\beta^{-1} \frac{\partial}{\partial k} \ln Z=-(\beta Z)^{-1} \frac{\partial Z}{\partial k} \ln Z \\
& =\frac{\int_{-\infty}^{\infty} \mathrm{d} \mathbf{r}^{d N} \frac{1}{2}\left|\mathbf{r}-\mathbf{r}_{0}\right|^{2} e^{-\beta k\left|\mathbf{r}-\mathbf{r}_{0}\right|^{2} / 2}}{\int_{-\infty}^{\infty} \mathrm{d} \mathbf{r}^{d N} e^{-\beta k\left|\mathbf{r}-\mathbf{r}_{0}\right|^{2} / 2}} \\
& =\frac{1}{2}\left\langle\left|\mathbf{r}-\mathbf{r}_{0}\right|^{2}\right\rangle,
\end{aligned}
$$

hence we can compute the mean squared distance for a $d N$-dimensional harmonic oscillator

$$
\left\langle\left|\mathbf{r}-\mathbf{r}_{0}\right|^{2}\right\rangle=2\left(\frac{\partial F(k)}{\partial k}\right)_{N V T}=\frac{d N}{\beta k} .
$$

For thermodynamic integration we are interested in the limit $k \rightarrow 0$. In this limit there is no penalty for moving the system as whole, hence the mean squared displacement becomes of the order of $L^{2}$, where $L$ is the box side length. This result means that the function $\left\langle\left|\mathbf{r}-\mathbf{r}_{0}\right|^{2}\right\rangle_{k}$ will be strongly peaked at $k=0$, thus making its integration difficult. For this reason, we would like this function to vary slowly with $k$. This behaviour can be achieved by fixing the centre of mass of the system, so that drifting as a whole is forbidden [107].

The centre of mass is defined as:

$$
\mathbf{r}_{\mathrm{CM}}=\sum_{i} \mu_{i} \mathbf{r}_{i}, \quad \text { where } \mu_{i}=\frac{m_{i}}{\sum_{i} m_{i}} .
$$

When computing the potential energy for the harmonic spring, we must apply the following correction:

$$
\left|\mathbf{r}^{(C)}-\mathbf{r}_{0}^{(C)}\right|^{2}=\sum_{i}^{N}\left|\mathbf{r}_{i}^{(U)}-\mathbf{r}_{i, 0}^{(U)}-\Delta \mathbf{r}_{i}^{(\mathrm{CM})}\right|^{2},
$$

where $i$ is the index for the $i$-th particle and $C$ and $U$ denote the corrected and the uncorrected coordinates respectively. The configurational partition function requires a correction, hence we define the corrected partition function $Z_{\mathrm{CM}}$ with centre of mass fixed at $\mathbf{r}_{\mathrm{CM}}=\mathbf{0}$ 
and note that:

$$
\begin{aligned}
Z_{\mathrm{CM}} & =\int_{-\infty}^{\infty} \mathrm{d} \mathbf{r}^{d N} e^{-\beta k\left|\mathbf{r}-\mathbf{r}_{0}\right|^{2} / 2} \delta\left(\sum_{i} \mu_{i} \mathbf{r}_{\mathbf{i}}\right) \\
& =\left(\frac{\beta k}{2 \pi \sum_{i} \mu_{i}^{2}}\right)^{d / 2}\left(\frac{2 \pi}{\beta k}\right)^{N d / 2}=\left(\frac{\beta k}{2 \pi \sum_{i} \mu_{i}^{2}}\right)^{d / 2} Z
\end{aligned}
$$

where solution of the integral was obtained after a fair amount of algebra by rewriting the Dirac delta as the Fourier $\operatorname{sum} \delta(\mathbf{x})=1 /\left(2 \pi^{3}\right) \int \mathrm{d} \mathbf{k} \exp (i \mathbf{k x})[103,118]$.

Using Eq. (D4) we find the mean squared displacement for the constrained Harmonic oscillator:

$$
\left\langle\left|\mathbf{r}-\mathbf{r}_{0}\right|^{2}\right\rangle_{\mathrm{CM}}=2\left(\frac{\partial F^{\mathrm{CM}}(k)}{\partial k}\right)_{N V T}=\frac{(N-1) d}{\beta k} .
$$

This result can be interpreted as the mean squared displacement of the $(N-1) d$ harmonic oscillator: fixing the centre of mass is equivalent to fixing one particle and integrating Eq. (D7) over the remaining degrees of freedom by doing the change of variables $\mathbf{r}_{i}^{\prime}=\mathbf{r}_{i}-\mathbf{r}_{N}$ (conveniently with unit Jacobian) if the $N$-th particle is fixed.

To conclude, let us relabel the potential as

$$
V\left(\mathbf{r} \mid \mathbf{r}_{0}, k, \lambda\right)=(1-\lambda) \Phi(\mathbf{r})+\frac{1}{2} \lambda k\left|\mathbf{r}-\mathbf{r}_{0}\right|^{2}
$$

where $\Phi(\mathbf{r})$ is an arbitrary field, it could be, for instance, an additional inter-atomic interaction independent of $k$ or, even the zero field. Let us consider the limit $\lambda \rightarrow 0$ : from the ratio of the partition functions for the constrained and unconstrained centre of mass, we find:

$$
\begin{aligned}
\frac{Z_{\mathrm{CM}}(\lambda=0)}{Z(\lambda=0)} & =\frac{\int_{-\infty}^{\infty} \mathrm{d} \mathbf{r}^{d N} e^{-\beta \Phi(\mathbf{r})} \delta\left(\sum_{i} \mu_{i} \mathbf{r}_{\mathbf{i}}\right)}{\int_{-\infty}^{\infty} \mathrm{d} \mathbf{r}^{d N} e^{-\beta \Phi(\mathbf{r})}} \\
& =\left\langle\delta\left(\sum_{i} \mu_{i} \mathbf{r}_{i}\right)\right\rangle=\mathcal{P}\left(\mathbf{r}_{\mathrm{CM}}=\mathbf{0}\right),
\end{aligned}
$$

where $\delta$ is the Dirac delta function and $\mathcal{P}\left(\mathbf{r}_{\mathrm{CM}}=0\right)$ is the probability density of the centre of mass being at $\mathbf{0}$ when $\lambda=0$. Hence we write:

$$
Z_{\mathrm{CM}}(\lambda=0)=Z(\lambda=0) \mathcal{P}\left(\mathbf{r}_{\mathrm{CM}}=0\right)
$$

where $\mathcal{P}$ depends on the details of the system. If the equilibrium structure is invariant to translations, a condition that holds true in a system with periodic boundary conditions, then we can take $\mathcal{P}=1 / V_{\text {cell }}$, where $V_{\text {cell }}$ is the smallest repeating unit in the periodic system (unit cell). This is at worst $V_{\text {cell }}=V_{\text {box }}$, while for a fcc Einstein crystal it would correspond to the Wigner-Seitz cell $V_{\text {cell }}=V_{\text {box }} / N[102]$.

\section{Appendix E: Polydisperse hard-sphere fluid and total accessible volume}

We can write the total accessible volume as

$$
-\ln \mathcal{V}(N, \phi)=-N \ln V_{\text {box }}+N f_{\text {ex }}(\phi),
$$

where $\phi$ is the volume fraction and $f_{\operatorname{ex}(\phi)}$ is the excess free energy, which is the difference in free energy between the hard sphere fluid and the ideal gas. We can compute the excess free energy by thermodynamic integration [107]. We start by noting that $\partial F / \partial\left(1 / V_{\text {box }}\right)=V_{\text {box }}^{2} P$ and define the number density $\rho=N / V_{\text {box }}$, hence we write

$$
f_{\mathrm{ex}}(\rho)=\frac{F(\rho)}{N}-\frac{F^{(\mathrm{id})}(\rho)}{N}=\int_{0}^{\rho} \mathrm{d} \rho^{\prime}\left(\frac{P\left(\rho^{\prime}\right)-\rho^{\prime}}{\rho^{\prime 2}}\right) .
$$

By noting that the volume fraction of a polydisperse system is $\phi=v_{d} \rho\left\langle\sigma^{d}\right\rangle$ [119], where $v_{d}$ is the volume of the $d$-dimensional unit sphere and $\left\langle\sigma^{d}\right\rangle$ is the $d$-th moment of the distribution of diameters, we can change variable and write

$$
f_{\mathrm{ex}}(\phi)=\frac{F(\phi)}{N}-\frac{F^{(\mathrm{id})}(\phi)}{N}=\int_{0}^{\phi} \mathrm{d} \phi^{\prime}\left(\frac{Z\left(\phi^{\prime}\right)-1}{\phi^{\prime}}\right),
$$

where $Z(\phi)=P / \rho$ is the compressibility factor (we set $\beta=1$ everywhere).

Analytical approximations for the compressibility factors for the two and three-dimensional polydisperse hard sphere fluid have been proposed. For the hard disk fluid we use the Santos-Yuste-Haro (eSYH) equation of state [119]

$$
\begin{aligned}
Z_{\mathrm{eSHY}}^{\mathrm{poly}}(\phi) & =\frac{\langle\sigma\rangle^{2} /\left\langle\sigma^{2}\right\rangle}{1-2 \phi+\left(2 \phi_{0}-1\right) \phi^{2} / \phi_{0}^{2}} \\
& +\frac{1}{1-\phi}\left(1-\frac{\langle\sigma\rangle^{2}}{\left\langle\sigma^{2}\right\rangle}\right),
\end{aligned}
$$
tion.

where $\phi_{0}=\pi / \sqrt{12}$ is the crystalline close packing frac-

For three-dimensional fluids, depending on the volume fraction, we choose two different equations of state. For volume fraction $\phi>0.5$, Santos et al. [119] suggest the following equation of state based on the CarnahanStartling (CS) equation of state for the monodisperse fluid:

$$
\begin{aligned}
Z_{\mathrm{eCS}}^{\mathrm{poly}}(\phi)=1+ & {\left[\frac{1+\phi+\phi^{2}-\phi^{3}}{(1-\phi)^{3}}-1\right] } \\
& \times \frac{\left\langle\sigma^{2}\right\rangle}{2\left\langle\sigma^{3}\right\rangle^{2}}\left(\left\langle\sigma^{2}\right\rangle^{2}+\langle\sigma\rangle\left\langle\sigma^{3}\right\rangle\right) \\
& +\frac{\phi}{1-\phi}\left[1-\frac{\left\langle\sigma^{2}\right\rangle^{2}}{\left\langle\sigma^{3}\right\rangle}\left(2\left\langle\sigma^{2}\right\rangle^{2}-\langle\sigma\rangle\left\langle\sigma^{3}\right\rangle\right)\right] .
\end{aligned}
$$


For volume fractions $\phi \leq 0.5$ the eCSK equation of state should be preferred (based on the CarnahanStarling-Kolafa equation of state for the monodisperse fluid)

$$
\begin{aligned}
Z_{\mathrm{eCSK}}^{\mathrm{poly}}(\phi) & =Z_{\mathrm{eCS}}^{\mathrm{poly}}(\phi) \\
& +\frac{\phi^{3}(1-2 \phi)}{(1-\phi)^{3}} \frac{\left\langle\sigma^{2}\right\rangle}{6\left\langle\sigma^{3}\right\rangle^{2}}\left(\left\langle\sigma^{2}\right\rangle^{2}+\langle\sigma\rangle\left\langle\sigma^{3}\right\rangle\right)
\end{aligned}
$$

The excess free energy can thus be obtained by substituting one of Eq. (E4) to (E6) in the integral of Eq. (E3), which can then be evaluated numerically for the desired volume fraction.

\section{Appendix F: Thermodynamic limit of the generalised configurational entropy}

From the fit to the empirical c.d.f. of $\mathcal{B}(\mathcal{P})$ with the generalised log-normal cumulative distribution function, corresponding to Eq. (28), we obtain the set of parameters $\mu, \sigma$, and $\zeta$. From the inset in Fig. 5, we observe that the mean $\mu$ and scale parameter $\sigma$ scale linearly with $1 / N$. In particular we note that $\sigma$ seems to approach zero in the thermodynamic limit, as expected. Furthermore we note that the shape parameter $\zeta$ seems to be approximately independent of $1 / N$ and to have a value of approximately 2 for all system sizes, thus suggesting that the distributions of pressures are consistent with a log-normal distribution.

Therefore, under the reasonable assumption that the biased distribution of pressures $\mathcal{B}(x \mid V)$ is $\log$-normal, we write the integrand in Eq. (11) as

$$
\begin{aligned}
& I(x ; \mu, \sigma, N) \equiv \mathcal{B}(x \mid V) x^{N / \kappa}= \\
& \frac{1}{x \sqrt{2 \pi \sigma^{2}}} \exp \left(-\frac{(\ln (x)-\mu)^{2}}{2 \sigma^{2}}+\frac{N}{\kappa} \ln (x)\right),
\end{aligned}
$$

which is a unimodal distribution with mode $x_{M}=$ $\exp \left(N \sigma^{2} / \kappa+\mu-\sigma^{2}\right)$. The distribution is such that

$$
\int_{0}^{\infty} I(x ; \mu, \sigma, N) \mathrm{d} x=\exp \left[\frac{\sigma^{2} N^{2}}{2 \kappa^{2}}+\frac{N \mu}{\kappa}\right] .
$$

Since $\sigma \propto 1 / N$, for large $N$ we have $\int_{0}^{\infty} I(x ; \mu, \sigma, N \gg$ $\kappa) \mathrm{d} x=\varepsilon \exp (N \mu / \kappa)$, with $\varepsilon$ some constant. Thus in the thermodynamic limit $(N, V, 1 / \sigma \rightarrow \infty)$ we obtain the expression for the Gibbs configurational entropy per particle, see Eq. (12).

Consider Eq. (6) which we rewrite in terms of the dimensionless free energy per particle

$$
f\left(\mathcal{P} \mid \phi_{\mathrm{SS}}\right) \equiv-\frac{1}{N} \ln (v)=c+\frac{1}{\kappa} \ln (\mathcal{P}),
$$

where $c=\mathcal{C}(N) / N$; to test the consistency of the results thus obtained we compare the expression for Edwards configurational entropy, see Eq. (12), to the Gibbs configurational entropy per particle, see Eq. (23). We thus find that

$$
\langle f\rangle_{\mathcal{B}}=c+\frac{1}{\kappa}\langle\ln (\mathcal{P})\rangle_{\mathcal{B}}
$$

which is consistent with Eq. (F3) in the thermodynamic limit. We have thus correctly recovered the power law relation between pressure and basin volume, Eq. (6).
[1] J. F. Kok, E. J. R. Parteli, T. I. Michaels, and D. B. Karam, "The physics of wind-blown sand and dust," Reports on Progress in Physics 75, 106901 (2012).

[2] M. Van Hecke, "Jamming of soft particles: geometry, mechanics, scaling and isostaticity," Journal of Physics: Condensed Matter 22, 033101 (2010).

[3] A. J. Liu and S. R. Nagel, "Nonlinear dynamics: Jamming is not just cool any more," Nature 396, 21 (1998).

[4] S. F. Edwards and R. B. S. Oakeshott, "Theory of powders," Physica A 157, 1080 (1989).

[5] H. A. Makse and J. Kurchan, "Testing the thermodynamic approach to granular matter with a numerical model of a decisive experiment," Nature 415, 614 (2002).

[6] C. Song, P. Wang, and H. A. Makse, "Experimental measurement of an effective temperature for jammed granular materials," Proceedings of the National Academy of Sciences of the United States of America 102, 2299 (2005).
[7] A. Barrat, J. Kurchan, V. Loreto, and M. Sellitto, 'Edwards' measures: a thermodynamic construction for dense granular media and glasses," Physical Review E 63, 051301 (2001).

[8] G.-J. Gao, J. Bławzdziewicz, and C. S. O'Hern, "Frequency distribution of mechanically stable disk packings," Physical Review E 74, 061304 (2006).

[9] F. Lechenault, F. da Cruz, O. Dauchot, and E. Bertin, "Free volume distributions and compactivity measurement in a bidimensional granular packing," Journal of Statistical Mechanics: Theory and Experiment, P07009 (2006).

[10] K. E. Daniels and N. W. Hayman, "Force chains in seismogenic faults visualized with photoelastic granular shear experiments," Journal of Geophysical Research: Solid Earth (1978-2012) 113, B11411 (2008).

[11] J. Brujić, C. Song, P. Wang, C. Briscoe, G. Marty, and H. A. Makse, "Measuring the coordination number and entropy of a $3 \mathrm{~d}$ jammed emulsion packing by con- 
focal microscopy," Physical Review Letters 98, 248001 (2007).

[12] V. Baranau, D. Hlushkou, S. Khirevich, and U. Tallarek, "Pore-size entropy of random hard-sphere packings," Soft Matter 9, 3361 (2013).

[13] E. R. Nowak, J. B. Knight, E. Ben-Naim, H. M. Jaeger, and S. R. Nagel, "Density fluctuations in vibrated granular materials," Physical Review E 57, 1971 (1998).

[14] M. Schröter, D. I. Goldman, and H. L. Swinney, "Stationary state volume fluctuations in a granular medium," Physical Review E 71, 030301 (2005).

[15] S. McNamara, P. Richard, S. K. De Richter, G. Le Caër, and R. Delannay, "Measurement of granular entropy," Physical Review E 80, 031301 (2009).

[16] D. S. Dean and A. Lefevre, "Possible test of the thermodynamic approach to granular media," Physical Review Letters 90, 198301 (2003).

[17] S.-C. Zhao, S. Sidle, H. L. Swinney, and M. Schröter, "Correlation between Voronoi volumes in disc packings," EPL (Europhysics Letters) 97, 34004 (2012).

[18] T. Aste and T. Di Matteo, "Emergence of gamma distributions in granular materials and packing models," Physical Review E 77, 021309 (2008).

[19] R. Blumenfeld and S. F. Edwards, "Granular entropy: Explicit calculations for planar assemblies," Physical Review Letters 90, 114303 (2003).

[20] R. Blumenfeld, J. F. Jordan, and S. F. Edwards, "Interdependence of the volume and stress ensembles and equipartition in statistical mechanics of granular systems," Physical Review Letters 109, 238001 (2012).

[21] S.-C. Zhao and M. Schröter, "Measuring the configurational temperature of a binary disc packing," Soft Matter 10, 4208 (2014)

[22] J. G. Puckett and K. E. Daniels, "Equilibrating temperaturelike variables in jammed granular subsystems," Physical Review Letters 110, 058001 (2013).

[23] D. Bi, S. Henkes, K. E. Daniels, and B. Chakraborty, "The statistical physics of athermal materials," Annual Review of Condensed Matter Physics 6, 63 (2015).

[24] C. Briscoe, C. Song, P. Wang, and H. A. Makse, "Entropy of jammed matter," Physical Review Letters 101, 188001 (2008).

[25] R. Blumenfeld and S. F. Edwards, "On granular stress statistics: Compactivity, angoricity, and some open issues," The Journal of Physical Chemistry B 113, 3981 (2009).

[26] S. Henkes, C. S. O'Hern, and B. Chakraborty, "Entropy and temperature of a static granular assembly: an ab initio approach," Physical Review Letters 99, 038002 (2007).

[27] S. Henkes and B. Chakraborty, "Statistical mechanics framework for static granular matter," Physical Review E 79, 061301 (2009).

[28] K. Wang, C. Song, P. Wang, and H. A. Makse, "Angoricity and compactivity describe the jamming transition in soft particulate matter," EPL (Europhysics Letters) 91, 68001 (2010).

[29] K. Wang, C. Song, P. Wang, and H. A. Makse, "Edwards thermodynamics of the jamming transition for frictionless packings: Ergodicity test and role of angoricity and compactivity," Physical Review E 86, 011305 (2012).

[30] J. H. Snoeijer, T. J. H. Vlugt, M. van Hecke, and W. van Saarloos, "Force network ensemble: a new ap- proach to static granular matter," Physical Review Letters 92, 054302 (2004).

[31] A. R. T. Van Eerd, B. P. Tighe, and T. J. H. Vlugt, "Numerical study of the force network ensemble," Molecular Simulation 35, 1168 (2009).

[32] B. P. Tighe, J. H. Snoeijer, T. J. H. Vlugt, and M. van Hecke, "The force network ensemble for granular packings," Soft Matter 6, 2908 (2010).

[33] B. P. Tighe and T. J. H. Vlugt, "Stress fluctuations in granular force networks," Journal of Statistical Mechanics: Theory and Experiment, P04002 (2011).

[34] D. Asenjo, F. Paillusson, and D. Frenkel, "Numerical calculation of granular entropy," Physical Review Letters 112, 098002 (2014).

[35] F. Paillusson, "Devising a protocol-related statistical mechanics framework for granular materials," Physical Review E 91, 012204 (2015).

[36] N. Xu, D. Frenkel, and A. J. Liu, "Direct determination of the size of basins of attraction of jammed solids," Physical Review Letters 106, 245502 (2011).

[37] M. Kac, "On the average number of real roots of a random algebraic equation (ii)," Proceedings of the London Mathematical Society 2, 390-408 (1948).

[38] K. Farahmand, "On the average number of real roots of a random algebraic equation," The Annals of Probability , 702-709 (1986).

[39] E. Bogomolny, O. Bohigas, and P. Leboeuf, "Distribution of roots of random polynomials," Physical Review Letters 68, 2726 (1992).

[40] A. Edelman and E. Kostlan, "How many zeros of a random polynomial are real?" Bulletin of the American Mathematical Society 32, 1-37 (1995).

[41] J. M. Rojas, "On the average number of real roots of certain random sparse polynomial systems," Lectures in Applied Mathematics-American Mathematical Society 32, 689-700 (1996)

[42] E. Kostlan, "On the expected number of real roots of a system of random polynomial equations," Foundations of Computational Mathematics , 149-188 (2000).

[43] G. Malajovich and J. M. Rojas, "High probability analysis of the condition number of sparse polynomial systems," Theoretical Computer Science 315, 525-555 (2004).

[44] J.-M. Azaïs and M. Wschebor, "On the roots of a random system of equations. the theorem of shub and smale and some extensions," Foundations of Computational Mathematics 5, 125-144 (2005).

[45] D. Armentano and M. Wschebor, "Random systems of polynomial equations. the expected number of roots under smooth analysis," Bernoulli 15, 249-266 (2009).

[46] Y. V. Fyodorov, G. A. Hiary, and J. P. Keating, "Freezing transition, characteristic polynomials of random matrices, and the riemann zeta function," Physical Review Letters 108, 170601 (2012).

[47] L. I. Nicolaescu, "Complexity of random smooth functions on compact manifolds. ii," arXiv:1209.0639 (2012).

[48] Y. V. Fyodorov, "High-dimensional random fields and random matrix theory," arXiv:1307.2379 (2013).

[49] D. Cheng and A. Schwartzman, "On the explicit height distribution and expected number of local maxima of isotropic gaussian random fields," arXiv:1503.01328 (2015).

[50] Y. V. Fyodorov, A. Lerario, and E. Lundberg, "On the 
number of connected components of random algebraic hypersurfaces," Journal of Geometry and Physics 95, $1-20(2015)$.

[51] Y. V. Fyodorov, "Complexity of random energy landscapes, glass transition, and absolute value of the spectral determinant of random matrices," Physical Review Letters 92, 240601 (2004).

[52] A. J. Bray and D. S. Dean, "Statistics of critical points of gaussian fields on large-dimensional spaces," Physical Review Letters 98, 150201 (2007).

[53] Y. V. Fyodorov and C. Nadal, "Critical behavior of the number of minima of a random landscape at the glass transition point and the tracy-widom distribution," Physical Review Letters 109, 167203 (2012).

[54] A. Auffinger, G. Ben Arous, and J. Černỳ, "Random matrices and complexity of spin glasses," Communications on Pure and Applied Mathematics 66, 165-201 (2013).

[55] Y. V. Fyodorov and P. Le Doussal, "Topology trivialization and large deviations for the minimum in the simplest random optimization," Journal of Statistical Physics 154, 466-490 (2014).

[56] C. Hughes, D. Mehta, and D. J. Wales, "An inversionrelaxation approach for sampling stationary points of spin model hamiltonians," The Journal of Chemical Physics 140, 194104 (2014).

[57] D. Mehta, T. Chen, J. D. Hauenstein, and D. J. Wales, "Communication: Newton homotopies for sampling stationary points of potential energy landscapes," The Journal of Chemical Physics 141, 121104 (2014).

[58] J. Cleveland, J. Dzugan, J. D. Hauenstein, I. Haywood, D. Mehta, A. Morse, L. Robol, and T. Schlenk, "Certified counting of roots of random univariate polynomials," arXiv:1412.1717 (2014).

[59] D. Mehta, M. Niemerg, and C. Sun, "Statistics of stationary points of random finite polynomial potentials," Journal of Statistical Mechanics: Theory and Experiment 2015, P09012 (2015).

[60] L. F. Cugliandolo and J. Kurchan, "Analytical solution of the off-equilibrium dynamics of a long-range spinglass model," Physical Review Letters 71, 173 (1993).

[61] J. Kurchan, G. Parisi, and M. A. Virasoro, "Barriers and metastable states as saddle points in the replica approach," Journal de Physique I 3, 1819-1838 (1993).

[62] D. Mehta, D. A. Stariolo, and M. Kastner, "Energy landscape of the finite-size mean-field 3-spin spherical model," Physical Review E 87, 052143 (2013).

[63] A. Auffinger and G. Ben Arous, "Complexity of random smooth functions on the high-dimensional sphere," The Annals of Probability 41, 4214-4247 (2013).

[64] E Subag, "The complexity of spherical p-spin models-a second moment approach," arXiv preprint arXiv:1504.02251 (2015).

[65] S. Sastry, "The relationship between fragility, configurational entropy and the potential energy landscape of glass-forming liquids," Nature 409, 164-167 (2001).

[66] A. Banerjee, S. Sengupta, S. Sastry, and S. M. Bhattacharyya, "Role of structure and entropy in determining differences in dynamics for glass formers with different interaction potentials," Physical Review Letters 113, 225701 (2014).

[67] J. Distler and U. Varadarajan, "Random polynomials and the friendly landscape," arXiv preprint hepth/0507090 (2005).
[68] M. R. Douglas and S. Kachru, "Flux compactification," Reviews of Modern Physics 79, 733-796 (2007).

[69] B. Greene, D. Kagan, A. Masoumi, D. Mehta, E. J. Weinberg, and X. Xiao, "Tumbling through a landscape: Evidence of instabilities in high-dimensional moduli spaces," Physical Review D 88, 026005 (2013).

[70] A. Aazami and R. Easther, "Cosmology from random multifield potentials," Journal of Cosmology and Astroparticle Physics 2006, 013 (2006).

[71] S.-H. H. Tye, J. Xu, and Y. Zhang, "Multi-field inflation with a random potential," Journal of Cosmology and Astroparticle Physics 2009, 018 (2009).

[72] J. Frazer and A. R. Liddle, "Exploring a string-like landscape," Journal of Cosmology and Astroparticle Physics 2011, 026 (2011).

[73] J. Frazer and A. R. Liddle, "Multi-field inflation with random potentials: field dimension, feature scale and non-gaussianity," Journal of Cosmology and Astroparticle Physics 2012, 039 (2012).

[74] D. Battefeld, T. Battefeld, and S. Schulz, "On the unlikeliness of multi-field inflation: bounded random potentials and our vacuum," Journal of Cosmology and Astroparticle Physics 2012, 034 (2012).

[75] G. Wainrib and J. Touboul, "Topological and dynamical complexity of random neural networks," Physical Review Letters 110, 118101 (2013).

[76] A. Choromanska, M. Henaff, M. Mathieu, G. Ben Arous, and Y. LeCun, "The loss surface of multilayer networks," arXiv:1412.0233 (2014).

[77] L. Sagun, V. U. Guney, and Y. LeCun, "Explorations on high dimensional landscapes," arXiv:1412.6615 (2014).

[78] P. Chaudhari and S. Soatto, "Trivializing the energy landscape of deep networks," arXiv:1511.06485 (2015).

[79] C. S. O'Hern, L. E. Silbert, A. J. Liu, and S. R. Nagel, "Jamming at zero temperature and zero applied stress: The epitome of disorder," Physical Review E 68, 011306 (2003).

[80] S. S. Ashwin, J. Bławzdziewicz, C. S. O'Hern, and M. D. Shattuck, "Calculations of the structure of basin volumes for mechanically stable packings," Physical Review E 85, 061307 (2012).

[81] D. Wales, Energy landscapes: Applications to clusters, biomolecules and glasses (Cambridge University Press, 2003).

[82] A. J. Ballard, S. Martiniani, J. D. Stevenson, S. Somani, and D. J. Wales, "Exploiting the potential energy landscape to sample free energy," Wiley Interdisciplinary Reviews: Computational Molecular Science 5, 273 (2015).

[83] D. Frenkel, D. Asenjo, and F. Paillusson, "The other entropy," Molecular Physics 111, 3641 (2013).

[84] R. J. Speedy, "Constrained simulations to count the glasses that a fluid samples," Journal of Molecular Structure 485-486, 537 (1999).

[85] D. J. Wales, "Surveying a complex potential energy landscape: Overcoming broken ergodicity using basinsampling," Chemical Physics Letters 584, 1 (2013).

[86] F. H. Stillinger and T. A. Weber, "Packing structures and transitions in liquids and solids," Science 225, 983 (1984).

[87] F. H. Stillinger and T. A. Weber, "Inherent structure theory of liquids in the hard-sphere limit," Journal of Chemical Physics 83, 4767 (1985). 
[88] F. H. Stillinger, "A topographic view of supercooled liquids and glass formation," Science 267, 1935 (1995).

[89] Results to appear elsewhere.

[90] B. D. Lubachevsky, F. H. Stillinger, and E. N. Pinson, "Disks vs. spheres: Contrasting properties of random packings," Journal of Statistical Physics 64, 501 (1991).

[91] S. Torquato, T. M. Truskett, and P. G. Debenedetti, "Is random close packing of spheres well defined?" Physical Review Letters 84, 2064 (2000).

[92] F. H. Stillinger and T. A. Weber, "Hidden structure in liquids," Physical Review A 25, 978 (1982).

[93] https://github.com/pele-python/mcpele.

[94] J. D. Weeks, D. Chandler, and H. C. Andersen, "Role of repulsive forces in determining the equilibrium structure of simple liquids," Journal of Chemical Physics 54, 5237 (1971).

[95] M. P. Allen and D. J. Tildesley, Computer simulation of liquids (Oxford university press, 1989).

[96] https://github.com/pele-python/pele.

[97] W. W. Hager and H. Zhang, "A new conjugate gradient method with guaranteed descent and an efficient line search," SIAM Journal on Optimization 16, 170 (2005).

[98] W. W. Hager and H. Zhang, "Algorithm 851: CG_DESCENT, a conjugate gradient method with guaranteed descent," ACM Transactions on Mathematical Software 32, 113 (2006).

[99] https://github.com/smcantab/PyCG_DESCENT.

[100] E. Bitzek, P. Koskinen, F. Gähler, M. Moseler, and P. Gumbsch, "Structural relaxation made simple," Physical Review Letters 97, 170201 (2006).

[101] P. G. Mezey, "Topology of energy hypersurfaces," Theoretica chimica acta 62, 133 (1982).

[102] D. Frenkel and A. J. C. Ladd, "New Monte Carlo method to compute the free energy of arbitrary solids. Application to the fcc and hcp phases of hard spheres," Journal of Chemical Physics 81, 3188 (1984).

[103] J. M. Polson, E. Trizac, S. Pronk, and D. Frenkel, "Finite-size corrections to the free energies of crystalline solids," Journal of Chemical Physics 112, 5339 (2000).

[104] A. P. Lyubartsev, A. A. Martsinovski, S. V. Shevkunov, and P. N. Vorontsov-Velyaminov, "New approach to Monte Carlo calculation of the free energy: Method of expanded ensembles," Journal of Chemical Physics 96, 1776 (1992).

[105] E. Marinari and G. Parisi, "Simulated tempering: a new Monte Carlo scheme," Europhysics Letters 19, 451 (1992).
[106] C. J. Geyer and E. A. Thompson, "Annealing Markov chain Monte Carlo with applications to ancestral inference," Journal of the American Statistical Association 90, 909 (1995).

[107] D. Frenkel and B. Smit, Understanding molecular simulation (Academic Press, San Diego, 2002).

[108] E. M. Knorr and R. T. Ng, "Algorithms for mining distance-based outliers in large datasets," in Proceedings of the 24rd International Conference on Very Large Data Bases (Morgan Kaufmann Publishers Inc., 1998) p. 392 .

[109] C. M. Bishop, Pattern recognition and machine learning (Springer, New York, 2009).

[110] http://scikit-learn.org/stable/modules/density. html.

[111] A. W. Bowman, "An alternative method of crossvalidation for the smoothing of density estimates," Biometrika 71, 353 (1984).

[112] B. Singh, K. K. Sharma, S. Rathi, and G. Singh, "A generalized log-normal distribution and its goodness of fit to censored data," Computational Statistics 27, 51 (2012).

[113] E. W. Weisstein, "Lobatto quadrature - from Wolfram MathWorld," http://mathworld.wolfram.com/ LobattoQuadrature.html.

[114] http://docs.scipy.org/doc/numpy/reference/ index.html.

[115] Q. Yan and J. J. de Pablo, "Hyper-parallel tempering Monte Carlo: Application to the Lennard-Jones fluid and the restricted primitive model," Journal of Chemical Physics 111, 9509 (1999).

[116] A. Bunker and B. Dünweg, "Parallel excluded volume tempering for polymer melts," Physical Review E 63, 016701 (2000).

[117] H. Fukunishi, O. Watanabe, and S. Takada, "On the Hamiltonian replica exchange method for efficient sampling of biomolecular systems: Application to protein structure prediction," Journal of Chemical Physics 116, 9058 (2002).

[118] C. Vega, E. Sanz, J. L. F. Abascal, and E. G. Noya, "Determination of phase diagrams via computer simulation: methodology and applications to water, electrolytes and proteins," Journal of Physics: Condensed Matter 20, 153101 (2008).

[119] A. Santos, S. B. Yuste, and M. López De Haro, "Equation of state of a multicomponent d-dimensional hardsphere fluid," Molecular Physics 96, 1 (1999). 